\title{
Solvable Critical Dense Polymers
}

\author{
Paul A. Pearce and Jørgen Rasmussen \\ Department of Mathematics and Statistics, University of Melbourne \\ Parkville, Victoria 3010, Australia \\ p.pearce@ms.unimelb.edu.au, j.rasmussen@ms.unimelb.edu.au
}

\begin{abstract}
A lattice model of critical dense polymers is solved exactly for finite strips. The model is the first member of the principal series of the recently introduced logarithmic minimal models. The key to the solution is a functional equation in the form of an inversion identity satisfied by the commuting double-row transfer matrices. This is established directly in the planar Temperley-Lieb algebra and holds independently of the space of link states on which the transfer matrices act. Different sectors are obtained by acting on link states with $s-1$ defects where $s=1,2,3, \ldots$ is an extended Kac label. The bulk and boundary free energies and finite-size corrections are obtained from the Euler-Maclaurin formula. The eigenvalues of the transfer matrix are classified by the physical combinatorics of the patterns of zeros in the complex spectral-parameter plane. This yields a selection rule for the physically relevant solutions to the inversion identity and explicit finitized characters for the associated quasi-rational representations. In particular, in the scaling limit, we confirm the central charge $c=-2$ and conformal weights $\Delta_{s}=\frac{(2-s)^{2}-1}{8}$ for $s=1,2,3, \ldots$. We also discuss a diagrammatic implementation of fusion and show with examples how indecomposable representations arise. We examine the structure of these representations and present a conjecture for the general fusion rules within our framework.
\end{abstract}




\section{Introduction}

Familiar materials such as plastics, nylon, polyester and plexiglass are made from polymers. Polymers consist of very long chain molecules with a large number of repeating structural units called monomers. Polymers exist in low- or high-temperature phases which are characterised as either dense or dilute. Polymers are dense if they fill a finite (non-zero) fraction of the available volume in the thermodynamic limit. In this paper, we are concerned with statistical models of dense polymers in two dimensions, both on the lattice and in the continuum. From the viewpoint of lattice statistical mechanics, polymers are of interest as prototypical examples of systems involving (extended) non-local degrees of freedom. It might be expected that the non-local nature of these degrees of freedom has a profound effect on the associated conformal field theory (CFT) obtained in the continuum scaling limit. Indeed, we confirm that the associated CFT is in fact logarithmic at least in the sense that certain representations of the dilatation Virasoro generator $L_{0}$ are non-diagonalizable and exhibit Jordan cells.

A simple model [1] of linear polymers, including excluded volume effects, is based on self-avoiding walks and can be obtained from the $O(n)$ model in the limit $n \rightarrow 0$. Similarly, a model [2] of branching polymers (spanning trees) is obtained from the $Q$-state Potts model, or more precisely the dichromatic polynomial, in the limit $Q \rightarrow 0$. Although these approaches [3, 4, 5, 6] have yielded much insight into polymers, including the central charge, conformal weights and conformal characters, they rely on the properties of analytic continuation in $n$ or $Q$.

In this paper, we propose a Yang-Baxter integrable model of critical dense polymers on the square lattice whose properties are directly accessible without the need for analytic continuation. In fact, this model is the first member $\mathcal{L} \mathcal{M}(1,2)$ of the principal series of the recently introduced integrable family of logarithmic minimal models [7]. We study the model on a finite-width strip subject to certain boundary conditions. Remarkably, as for the square lattice Ising model, the transfer matrices of this model satisfy a single functional equation in the form of an inversion identity which is independent of the boundary conditions. This enables us to obtain the exact properties of the model on a finite lattice. The conformal properties are then readily accessible from finite-size corrections. In particular, in the continuum scaling limit, we confirm the central charge $c=-2$ and conformal weights $\Delta_{s}=\frac{(2-s)^{2}-1}{8}$ where the extended Kac label $s=1,2,3, \ldots$ labels the boundary condition. Although all of these representations appear to be diagonalizable, we show how indecomposable representations are obtained by fusing boundary conditions.

In contrast to simple rational CFTs, in the case of logarithmic CFTs, there can be many different models with the same central charge and conformal weights. Specifically, the logarithmic theories at $c=-2$ include Hamiltonian walks on a Manhattan lattice [8, 9], the rational triplet theory [10, 11, 12], symplectic fermions [13], the Abelian sandpile model [14], dimers [15], the travelling salesman problem [16] as well as branching polymers [17]. Although much is known about these models, it seems fair to say that at 
present the precise relation between these various models is still not clear.

The layout of this paper is as follows. In Section 2, we define our model of critical dense polymers and the planar Temperley-Lieb (TL) algebra [18] on which it is built. In Section 3, we set up double-row transfer matrices and prove the inversion identity directly in the planar TL algebra. We define vector spaces of link states on which the transfer matrices act and relate these to the boundary conditions. We solve the inversion identity to obtain the general solution for the eigenvalues of the transfer matrices. The physically relevant solutions, in the sectors with $\ell=s-1$ defects, are obtained empirically by selection rules encoded in suitably defined double-column diagrams. In Section 4, the finite-size corrections to the eigenvalues are obtained by applying the Euler-Maclaurin formula yielding the bulk and boundary free energies and confirming the central charge $c=-2$ and conformal weights $\Delta_{s}=\frac{(2-s)^{2}-1}{8}$. In Section 5 , the finitized conformal characters for $s=1,2,3, \ldots$ are obtained explicitly from finite-width partition functions on the strip. It is also verified that these yield the quasi-rational characters of [7] in the continuum scaling limit. We discuss the Hamiltonian limit of the double-row transfer matrix in Section 6. In Section 7, we consider indecomposable representations. While it is well known [19] that the TL algebra admits indecomposable representations at roots of unity, this abstract result gives no hint as to how to construct these representations on the lattice or to relate them to physical models with physical boundary conditions. Here we exhibit the indecomposable representations of the dilatation generator $L_{0}$ arising from the lattice Hamiltonian and discuss how the fusion of such representations is implemented in terms of boundary conditions. The lattice approach to fusion is well established [20] in the context of boundary rational CFT and we extend the same principles here to logarithmic CFT. We conclude with some remarks and directions for future research in Section 8.

\section{Lattice Model}

\subsection{Critical dense polymers}

To model critical dense polymers on a finite strip, consider a square lattice consisting of $N$ columns and $N^{\prime}$ rows of faces. An elementary face of the lattice can assume one of two configurations with different statistical weights

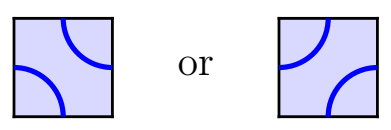

where the arcs represent local segments of polymers. Since the polymer segments pass uniformly through each face, this is a model of dense polymers. A typical configuration 
looks like

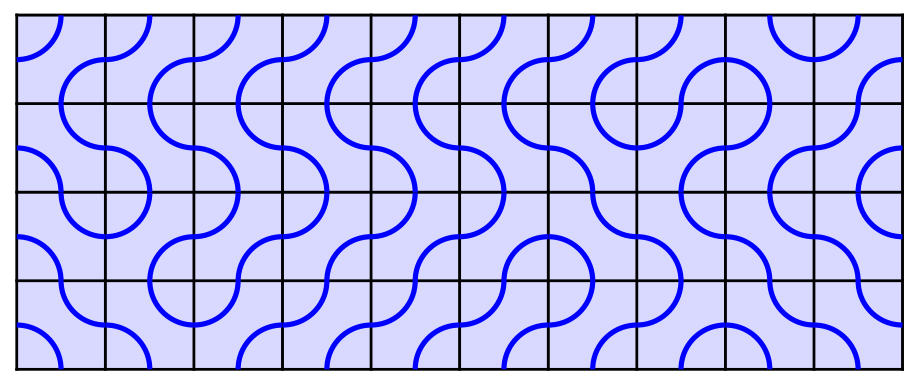

Once the boundary conditions have been specified, one is left with non-local degrees of freedom corresponding to a number of polymers. It is often convenient to think of these degrees of freedom as non-local connectivities. Since polymers are not allowed to close on themselves, the fugacity $\beta$ assigned to loops in the associated loop gas vanishes.

Critical dense polymers, as defined above, corresponds to the first member $\mathcal{L} \mathcal{M}(1,2)$ of the infinite principal series $\mathcal{L} \mathcal{M}(m, m+1)$ of logarithmic minimal models introduced in [7]. Each logarithmic model is characterized by a crossing parameter $\lambda$ which is a rational multiple of $\pi$ related to the loop fugacity $\beta$ by

$$
\beta=2 \cos \lambda
$$

It was argued in [7] that the scaling limits of these integrable lattice models yield logarithmic CFTs. In the case of critical dense polymers,

$$
\lambda=\frac{\pi}{2}
$$

implying that loops appear with weight zero which is equivalent to simply disallowing loops.

In this paper, we solve exactly our model of critical dense polymers on a strip. Remarkably, we can solve our model for arbitrary finite sizes. The only other known case where this is possible is the Ising model on the square lattice [21, 22]. The key to our solution is a functional equation in the form of an inversion identity reminiscent of the Ising model. This inversion identity is established directly in the planar Temperley-Lieb (TL) algebra [18. As will be explained in Section 3, it therefore holds independently of the set of link states on which it acts, that is, for all matrix representations of the double-row transfer matrix.

\subsection{Planar Temperley-Lieb algebra}

To fix notation, we review here the basics of the planar TL algebra. We refer to [18, 7] and references therein for more details.

An elementary face of the square lattice is assigned a face weight according to the configuration of the face. The weights for the two possible configurations can be combined 
into a single face weight as

$$
u=\sin u \square+\cos u \square
$$

where the lower left corner has been marked to fix the orientation of the square.

For the purposes of this paper, the planar TL algebra is a diagrammatic algebra built up from elementary faces. The faces are connected such that the midpoint of an outer edge of a face, called a node, can be linked to a node of any other (or even the same) face as long as the total set of links make up a non-intersecting planar web of connections. Two basic local properties of the planar TL algebra are the inversion relation

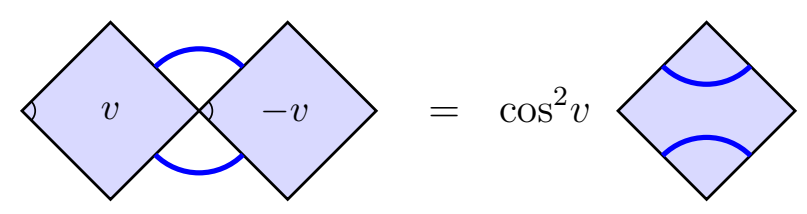

and the Yang-Baxter equation (YBE) [21]

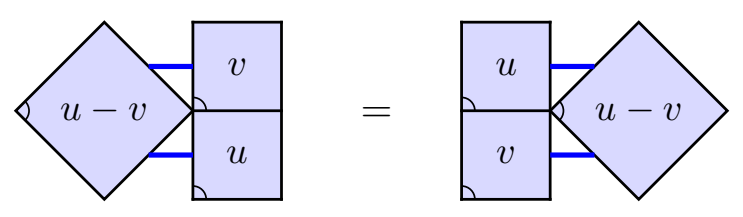

These are identities for 2- and 3-tangles, respectively, where a $k$-tangle is an arrangement of faces with $2 k$ free nodes. The identities are established by writing out all the possible configurations, while keeping track of the associated weights, and collecting them in classes according to connectivities. The left side of (2.6), for example, thus corresponds to a sum of four terms of which one vanishes since $\beta=0$. The remaining three terms fall into the two connectivity classes

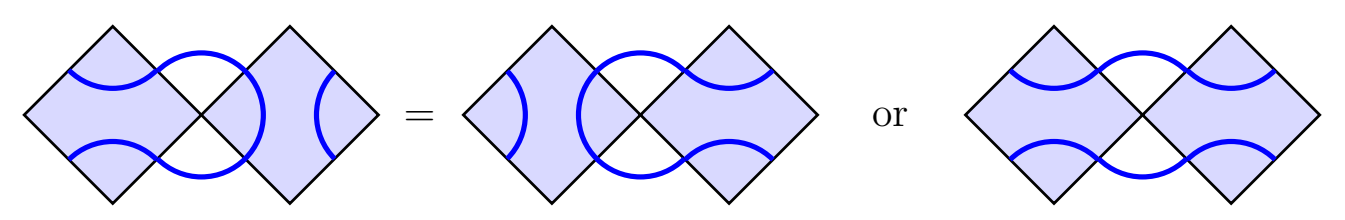

The weights accompanying the two equivalent configurations cancel since $\cos v \sin (-v)+$ $\sin v \cos (-v)=0$, while the last diagram comes with the weight $\cos v \cos (-v)$ thereby yielding the identity (2.6). 


\section{Solution on a Finite Strip}

\subsection{Double-row transfer matrix}

Having introduced the planar TL algebra, we define diagrammatically the double-row $N$-tangle

$$
\boldsymbol{D}(u)=\frac{1}{\sin 2 u} \begin{array}{|c|c|c|c|c|c|c|c|}
\hline \frac{\pi}{2}-u & & \cdots & & & \cdots & & \frac{\pi}{2}-u \\
\hline u & & \cdots & & & \cdots & & u \\
\hline
\end{array}
$$

consisting of $N$ two-columns, but suppress the dependence on $N$. As discussed below, $\boldsymbol{D}(u)$ has a natural matrix representation when acting vertically from below on a given set of so-called link states. We thus refer to it as the double-row transfer matrix, even though it is defined as a planar $N$-tangle without reference to any matrix representation. The normalization in (3.1) ensures that

$$
\begin{array}{l|l|l|l|l|l|l|l|l|l|}
\lim _{u \rightarrow 0} \boldsymbol{D}(u)=\boldsymbol{I}= & & & & & & & & \\
\hline
\end{array}
$$

where $\boldsymbol{I}$ is the (vertical) identity operator linking every node on the upper horizontal edge to the node directly below it on the lower horizontal edge. Aside from the normalization, the double-row transfer matrix $\boldsymbol{D}(u)$ is equivalent to the double-row transfer matrices introduced in [7] for general fugacity $\beta$. Using diagrammatic arguments based on [23], it follows that $\boldsymbol{D}(u)$ is crossing symmetric

$$
\boldsymbol{D}\left(\frac{\pi}{2}-u\right)=\boldsymbol{D}(u)
$$

and that it gives rise to a commuting family of transfer matrices where $[\boldsymbol{D}(u), \boldsymbol{D}(v)]=0$. Here the implied multiplication means vertical concatenation of the two $N$-tangles in the planar TL algebra.

\subsection{Inversion identity}

Remarkably, the double-row transfer matrix (3.1) satisfies an inversion identity in the planar TL algebra. This simple inversion identity is unique to critical dense polymers among the logarithmic minimal models [7].

Inversion identity The planar $N$-tangle $\boldsymbol{D}(u)$ defined in (3.1) satisfies the inversion identity

$$
\boldsymbol{D}(u) \boldsymbol{D}\left(u+\frac{\pi}{2}\right)=\left(\frac{\cos ^{2 N} u-\sin ^{2 N} u}{\cos ^{2} u-\sin ^{2} u}\right)^{2} \boldsymbol{I}
$$


where $\boldsymbol{I}$ is the identity $N$-tangle (3.2).

Proof of inversion identity The left side of (3.4) corresponds diagrammatically to

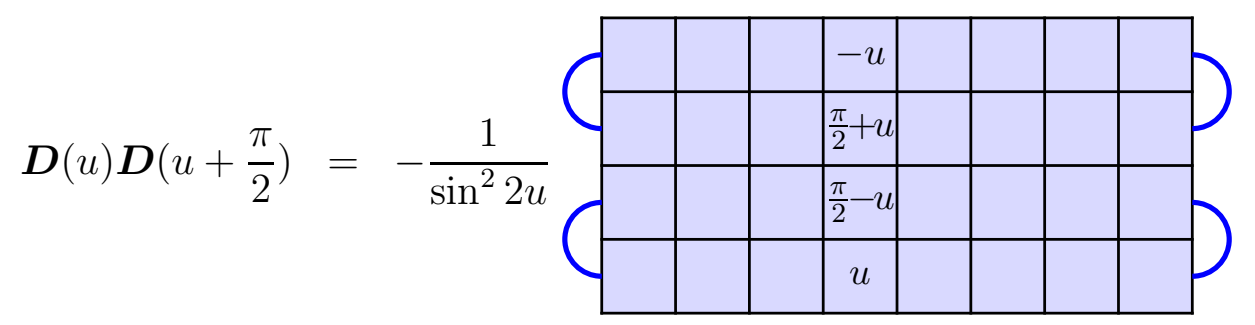

where all faces have the standard orientation, cf. (2.5) and (3.1). Employing the local inversion relation (2.6) once followed by repeated applications of the push-through property of the YBE (2.7), transforms the right side of (3.5) to
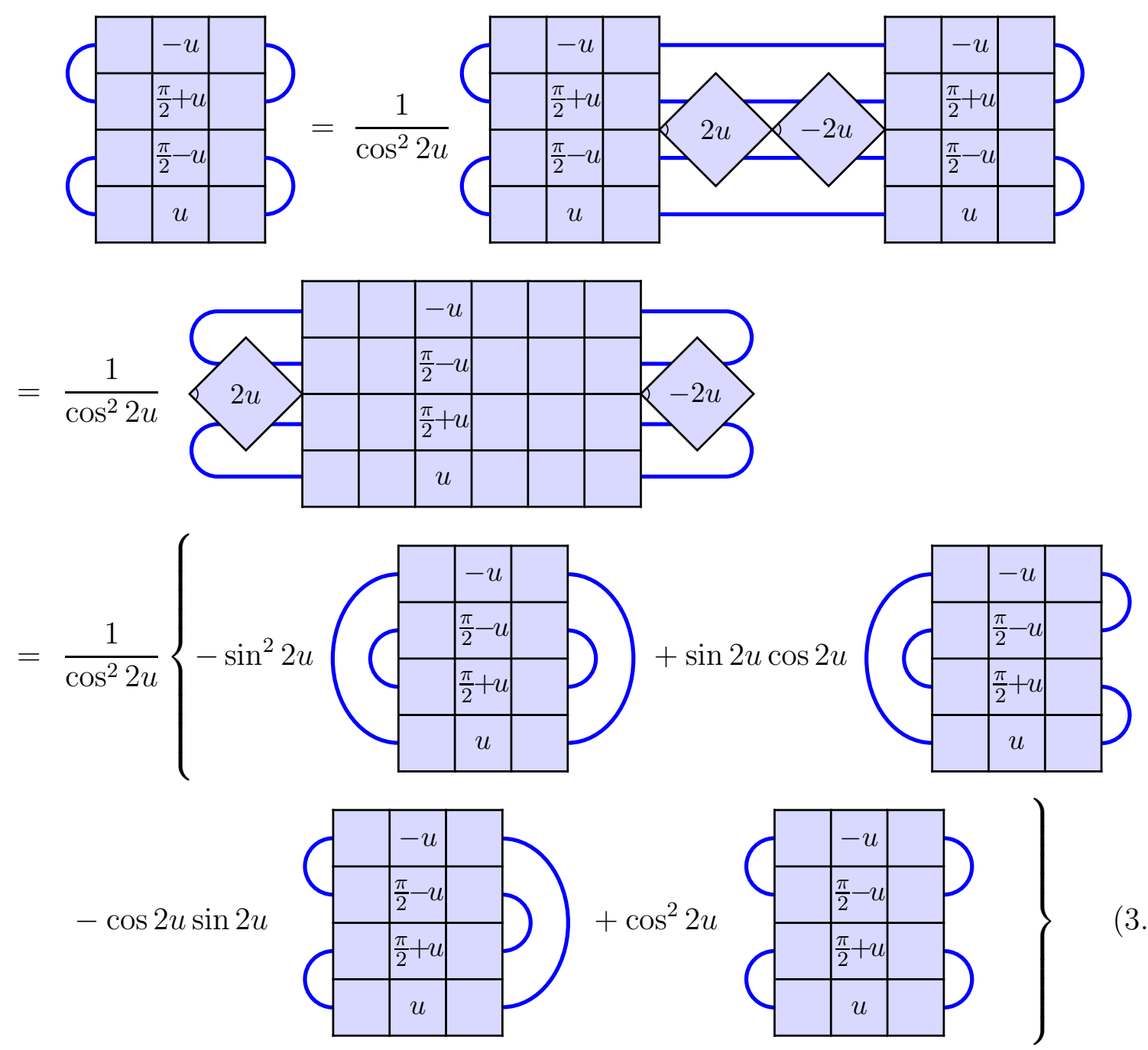
The last equality follows from an expansion in terms of connectivities of the two faces with face weights $\pm 2 u$. We now eliminate the last three diagrams by examining the consequences of having a half-arc connecting the two left edges (or two right edges) of a two-column appearing in the two lower or two upper rows. Expanding in terms of connectivities, we find

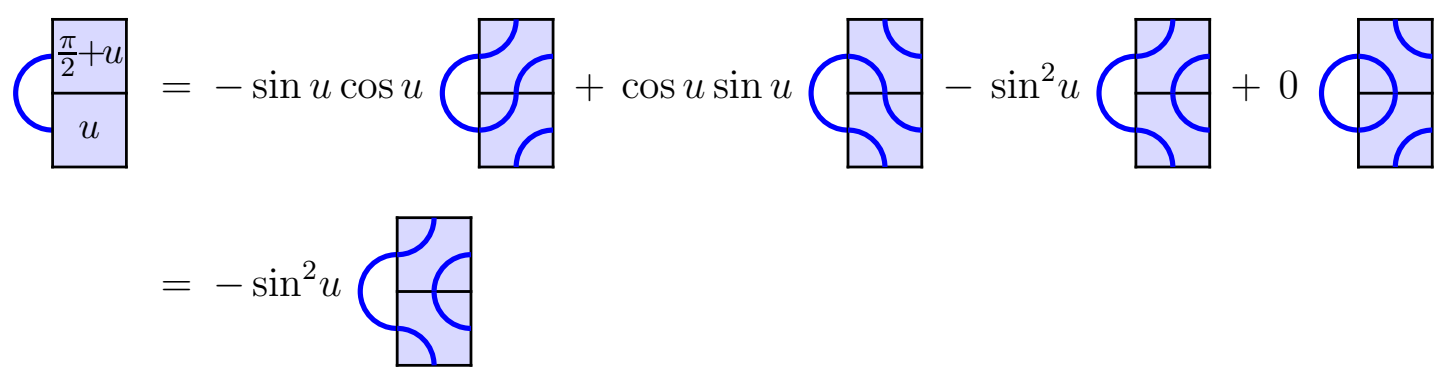

and similarly

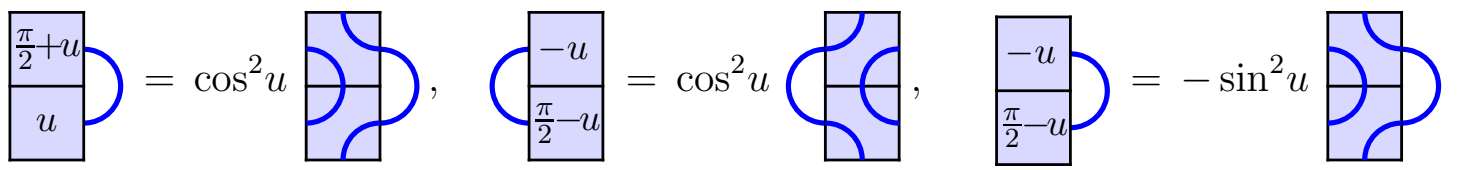

We observe that such a half-arc will propagate. In all three diagrams under consideration (3.6), this will eventually lead to a closed loop thus yielding a vanishing contribution. Also due to the propagating property of the half-arcs, the surviving diagram on the right 
side of (3.6) may be expanded as
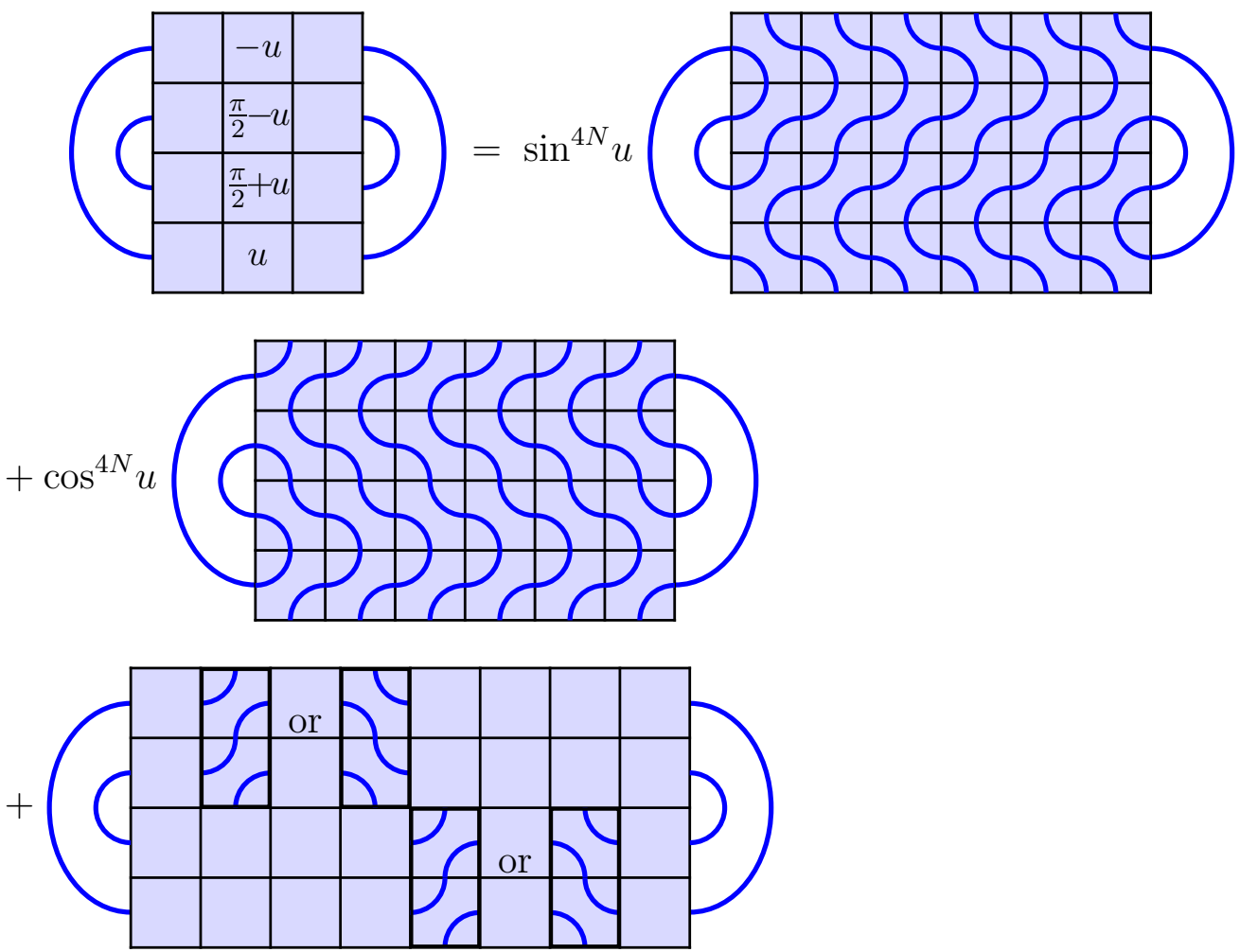

There are two possible connectivities in the last diagram for each two-column configuration in the lower or upper half, respectively. Since the two first diagrams in (3.9) both represent the identity (3.2), we now focus on the last diagram. Any given four-column configuration in this diagram can have only one of four possible connectivities. Reading from the left, we find by expanding in terms of these connectivities that

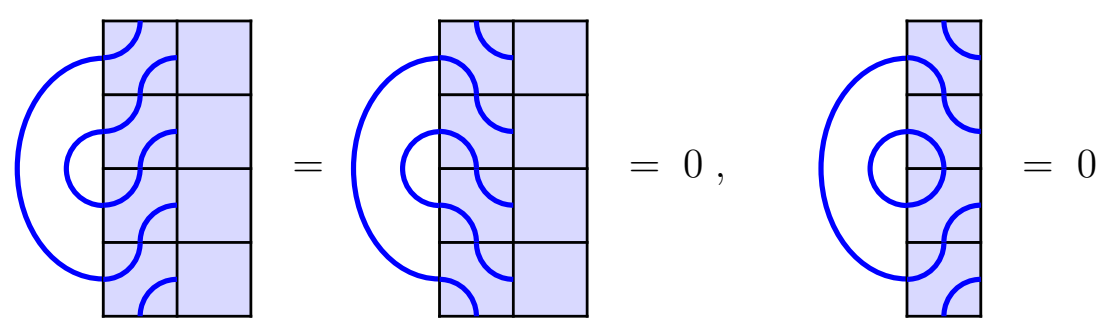

This implies that a pair of nested half-arcs, as in the last diagram of (3.9), will propagate towards the right. The pair will not, however, propagate all the way to the right to form a pair of closed loops since the sums over possible connectivities of the second four-columns were required to establish the vanishing of the left diagrams in (3.10). Continuing the 
evaluation in (3.9), we thus have

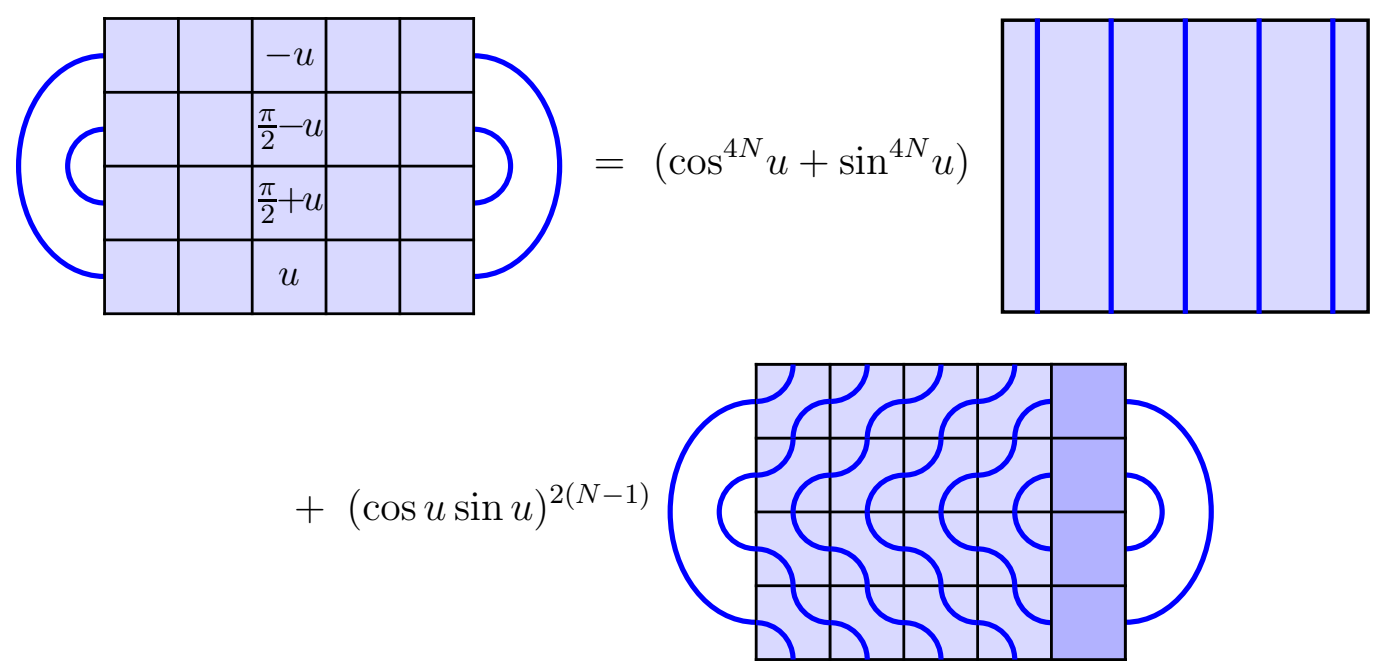

where

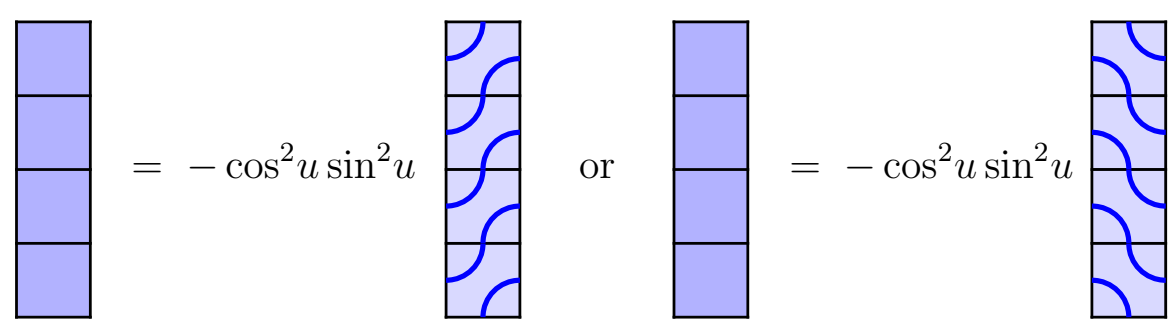

Combining the weights following from the various steps in this analysis readily produces (3.4) thus completing the proof.

It is noted that one could have read the last diagram in (3.9) from the right instead which would have involved identities obtained from (3.10) by reflection with respect to a vertical line. In this case, the four-column (3.12) would have appeared as the first four-column in the last diagram in (3.11) while the conclusion (3.4) remains the same.

\subsection{Link states}

A family, labelled by $\ell$, of matrix representations of $\boldsymbol{D}(u)$ is obtained by acting with $\boldsymbol{D}(u)$ from below on link states with precisely $\ell$ defects. It is recalled that $\boldsymbol{D}(u)$ has $N$ free nodes on the upper horizontal edge. A link state specifies how these $N$ nodes are linked together. A node that is not linked to another node gives rise to a defect which may be viewed as a link to the point (above) at infinity. The following represents a link state with 11 nodes and three defects

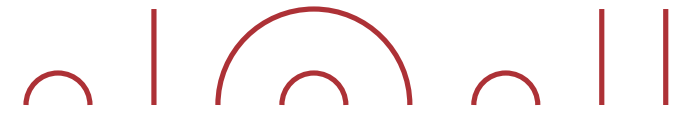


Let $\mathcal{L}_{N, \ell}$ denote the vector space of link states with precisely $\ell$ defects. Its dimension is

$$
\operatorname{dim}\left(\mathcal{L}_{N, \ell}\right)=\left(\begin{array}{c}
N \\
\frac{N-\ell}{2}
\end{array}\right)-\left(\begin{array}{c}
N \\
\frac{N-\ell-2}{2}
\end{array}\right)
$$

If the action of the planar TL algebra is unrestricted, then defects can be annihilated in pairs
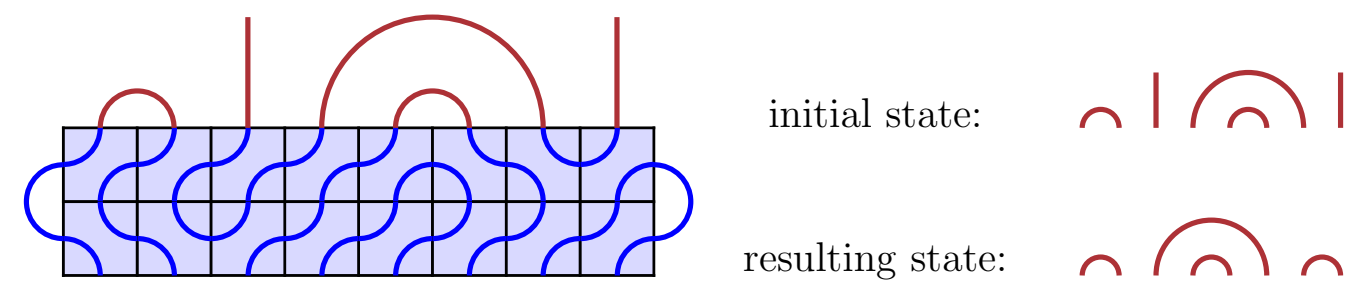

To associate a fixed number of defects $\ell$ with a boundary condition on the right (or left), we can close the defects on the right (or left) and allow them to propagate down the edge of the strip. In this case, the action of $\boldsymbol{D}(u)$ is restricted to $\mathcal{L}_{N, \ell}$ by forbidding annihilations of any pair of defects. The following illustrates this action of $\boldsymbol{D}(u)$ on link states with $\ell=2$ defects for a particular configuration with $N=8$

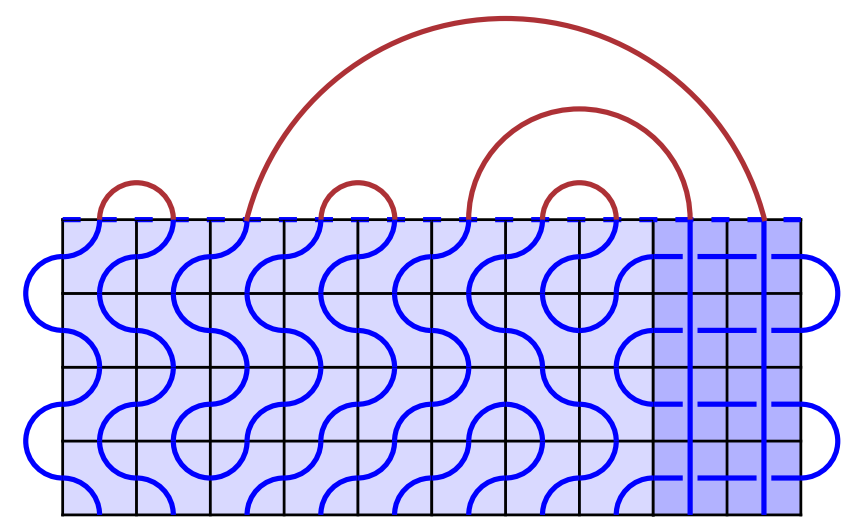

We label this boundary condition by $s=\ell+1=1,2,3, \ldots$ and refer to this restricted action of $\boldsymbol{D}(u)$ as a block or sector even though $\ell$ is not a quantum number. In general, these blocks are not normal, not symmetric and may have repeated eigenvalues. We have nevertheless observed and verified in many examples that this restricted action of $\boldsymbol{D}(u)$ is diagonalizable. This is in contrast to the unrestricted action of $\boldsymbol{D}(u)$ which in general is non-diagonalizable. We will return to this issue when discussing fusion in Section 7.

\subsection{Exact solution for the eigenvalues}

Once a matrix representation of $\boldsymbol{D}(u)$ has been fixed, the planar inversion identity (3.4) for the specified representation translates into a functional relation satisfied by the accompanying eigenvalues $D(u)$. It reads

$$
D(u) D\left(u+\frac{\pi}{2}\right)=\left(\frac{\cos ^{2 N} u-\sin ^{2 N} u}{\cos ^{2} u-\sin ^{2} u}\right)^{2}
$$


where the normalization (3.2) and the crossing symmetry (3.3) yield the conditions

$$
D(0)=1, \quad D\left(\frac{\pi}{2}-u\right)=D(u)
$$

The analysis of (3.17) depends on the parity of $N$ as we have the factorization

$$
\frac{\cos ^{2 N} u-\sin ^{2 N} u}{\cos ^{2} u-\sin ^{2} u}= \begin{cases}\frac{N}{2^{N-1}} \prod_{j=1}^{\frac{N}{2}-1}\left(\frac{1}{\sin ^{2} \frac{j \pi}{N}}-\sin ^{2} 2 u\right), & N \text { even } \\ \frac{1}{2^{N-1}} \prod_{j=1}^{\frac{N-1}{2}}\left(\frac{1}{\sin ^{2} \frac{(2 j-1) \pi}{2 N}}-\sin ^{2} 2 u\right), & N \text { odd }\end{cases}
$$

It is now straightforward to solve the functional relation (3.17), subject to (3.18), by sharing out the zeros. We find

$$
D(u)= \begin{cases}\frac{N}{2^{N-1}} \prod_{j=1}^{\frac{N}{2}-1}\left(\frac{1}{\sin \frac{j \pi}{N}}+\epsilon_{j} \sin 2 u\right)\left(\frac{1}{\sin \frac{j \pi}{N}}+\mu_{j} \sin 2 u\right), & N \text { even } \\ \frac{1}{2^{N-1}} \prod_{j=1}^{\frac{N-1}{2}}\left(\frac{1}{\sin \frac{(2 j-1) \pi}{2 N}}+\epsilon_{j} \sin 2 u\right)\left(\frac{1}{\sin \frac{(2 j-1) \pi}{2 N}}+\mu_{j} \sin 2 u\right), & N \text { odd }\end{cases}
$$

where $\epsilon_{j}^{2}=\mu_{j}^{2}=1$ for all $j$. The appearance of two sets of parameters $\left\{\epsilon_{j}\right\}$ and $\left\{\mu_{j}\right\}$ stems from the overall squaring in (3.17).

For either parity of $N$ in (3.20), the maximum eigenvalue is obtained for $\epsilon_{j}=\mu_{j}=1$ for all $j$ and corresponds to the ground state in the associated sector. Excited states are generated by switching a (finite) number of the parameters $\epsilon_{j}, \mu_{j}$ from 1 to -1 . The number of possible excitations following from (3.20) is $2^{N-2}$ and $2^{N-1}$, respectively, clearly exceeding the number of link states with a definite number of defects, cf. (3.14). We thus need a set of selection rules to determine which eigenvalues actually appear in the spectrum and therefore are physical. This information is neatly encoded by specifying the patterns of complex zeros of $D(u)$ which are allowed.

Generally, the zeros of an eigenvalue $D(u)$ come in conjugate pairs in the complex $u$-plane and appear with a periodicity $\pi$ in the real part of $u$. They follow from (3.20) and are collectively described by

$$
u \in\left\{\left(2+\nu_{j}\right) \frac{\pi}{4} \pm \frac{i}{2} \ln \tan \frac{t_{j}}{2}\right\}+\pi \mathbb{Z}
$$

where $\nu_{j}$ is $\epsilon_{j}$ or $\mu_{j}$, and where we have introduced the arguments

$$
t_{j}= \begin{cases}\frac{j \pi}{N}, & N \text { even } \\ \frac{(2 j-1) \pi}{2 N}, & N \text { odd }\end{cases}
$$


A typical pattern for $N=12$ is

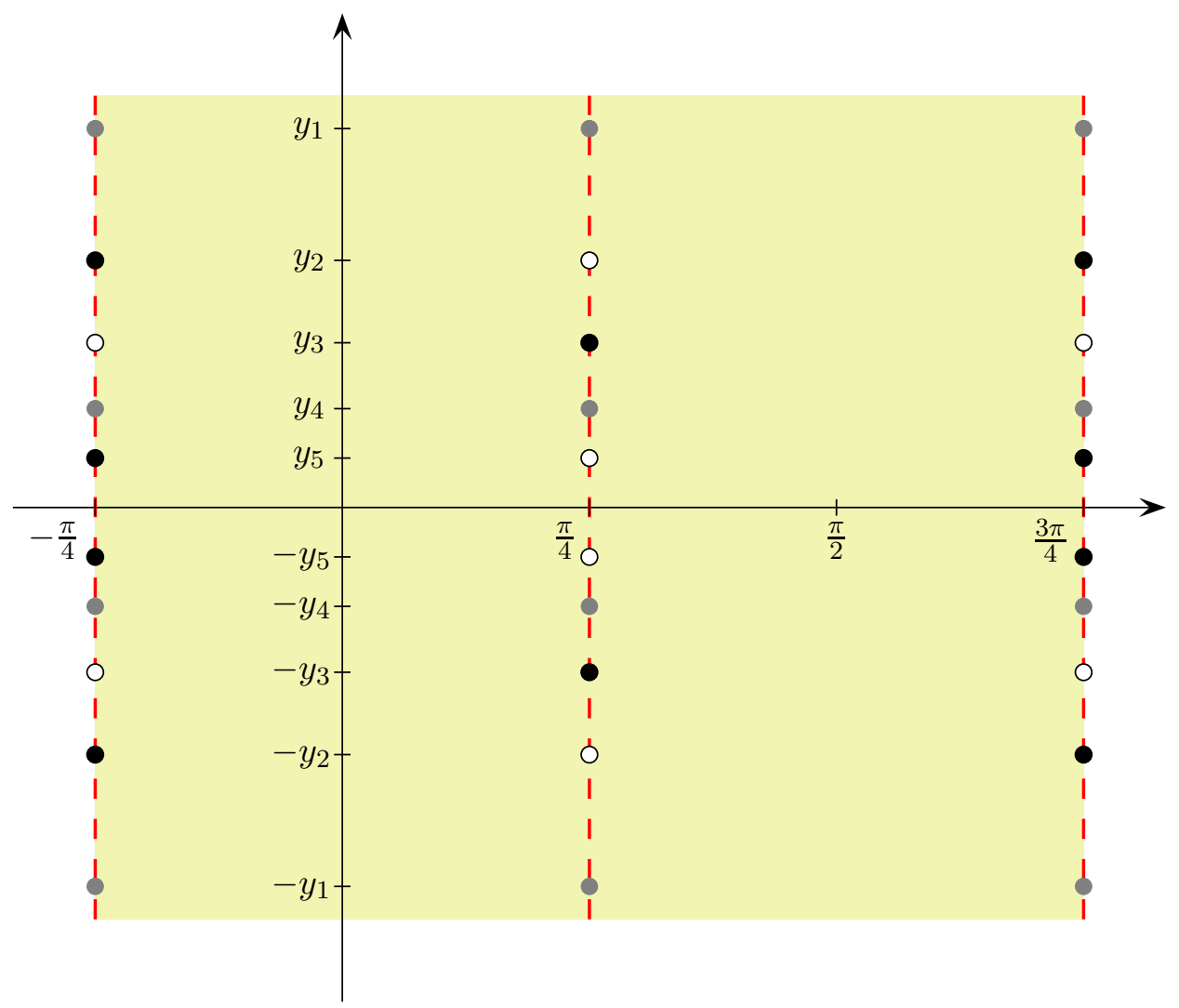

where

$$
y_{j}=-\frac{i}{2} \ln \tan \frac{t_{j}}{2}
$$

The infinite analyticity strip bounded by $u=-\frac{\pi}{4}$ and $u=\frac{3 \pi}{4}$ is called the physical strip. All zeros in the strip lie either on the boundary or on the vertical centre line (1-strings). In contrast to the usual situation in the context of Bethe ansatz, these zeros can occur either as single or double zeros. A single zero is indicated by a grey dot while a double zero is indicated by a black dot. Counting a double zero twice, the number of zeros with fixed imaginary value $\pm y_{j}$ and real part either $\frac{\pi}{4}$ or $\frac{3 \pi}{4}$ is two. This follows straightforwardly from (3.21) since $\nu_{j}$ can be either $\epsilon_{j}$ or $\mu_{j}$, as depicted in (3.23). The fact that the zeros appear in complex conjugate pairs is now seen to be a consequence of the crossing symmetry (3.18) and the periodicity in the $u$-plane.

It follows that the full pattern of zeros is encoded in the distribution of 1-strings in the lower (or equivalently upper) half-plane. This distribution is actually a sum of two, one governed by excitations administered by $\epsilon$ and one by $\mu$. The separation of the 1-strings into contributions coming from $\epsilon$ and $\mu$, respectively, is superfluous but nonetheless a 
helpful refinement later for the description of the selection rules. It is illustrated here

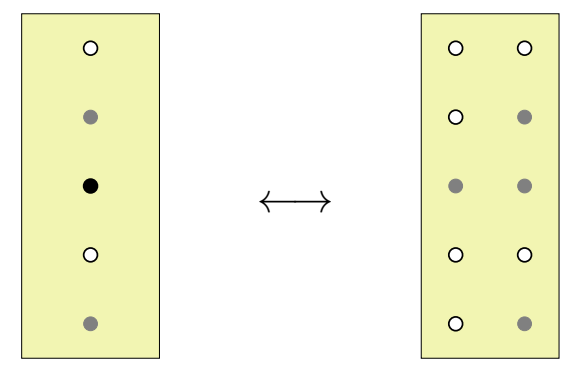

where the left side corresponds to (3.23) while the two columns to the right encode separately the $\epsilon$ and $\mu$ excitations. Not all such double-column configurations will appear. A characterization of a set of admissible two-column configurations provides a description of the selection rules. This combinatorial designation of the physical states is termed 'physical combinatorics'. The details of the combinatorial content is the topic of our forthcoming paper [24]. A synopsis follows below and is used in our description of the selection rules to be discussed subsequently. As we will see, the double-column configurations also provide a natural basis for defining the associated finitized characters.

\subsection{Double-column configurations}

A single-column configuration of height $M$ consists of $M$ sites arranged as a column. The sites are labelled from the bottom by the integers $1, \ldots, M$ and are weighted accordingly by $1, \ldots, M$. A site can be occupied or unoccupied. The signature $S$ of a single-column configuration is constructed as the set of weights of the occupied sites in the configuration listed in descending order. The weight $w$ of a configuration is given by the sum of the signature entries

$$
w(S)=\sum_{j} S_{j}
$$

A double-column configuration of height $M$ consists of a pair of single-column configurations of height $M$. The notions of signature and weight are readily extended from the single-column case as illustrated by the right side of (3.25). It has weight $w=11$ while the signature is

$$
S=(L, R), \quad L=(3), \quad R=(4,3,1)
$$

Here $L$ and $R$ refer to the signatures of the left and right single-column configurations, respectively. With $m$ and $n$ indicating the number of occupied sites in the left and right columns, respectively, the weight $w$ of a double-column configuration is given by

$$
w(L, R)=\sum_{j=1}^{m} L_{j}+\sum_{j=1}^{n} R_{j}
$$

The set of single-column configurations of height $M$ admits the partial ordering

$$
S \preceq S^{\prime} \quad \text { if } \quad S_{j} \leq S_{j}^{\prime}, \quad j=1, \ldots, m
$$


where $S$ and $S^{\prime}$ are two such single-column configurations with $m$ and $m^{\prime}$ occupied sites, respectively. A double-column configuration with signature $S=(L, R)$ is called admissible if $L \preceq R$. This presupposes, in particular, that

$$
0 \leq m \leq n \leq M
$$

The configuration (3.25) is seen to be admissible. This convention for admissibility corresponds to $\mu$ being dominant over $\epsilon$. We let $A_{m, n}^{M}$ denote the set of admissible doublecolumn configurations with $m$ occupied sites in the left column and $n$ occupied sites in the right column. It is noted that the set $A_{m, n}^{M}$ is empty if and only if one of the inequalities (3.30) is violated.

There is a very simple 'geometric' characterization of admissible double-column configurations. As in the example

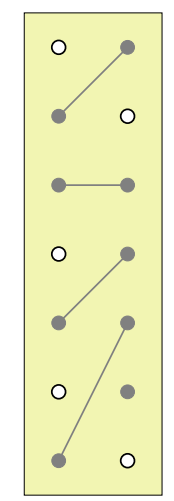

one draws line segments between the occupied sites of greatest weight in the two columns, between the occupied sites of second-to-greatest weight etc. Admissible double-column configurations are now characterized by not involving line segments with a negative slope. It could involve no line segments $(m=0)$, while a line segment can only appear with non-negative slope. The example (3.31) is immediately recognized as being admissible. As illustrated in (3.31), grey dots ultimately not linked to any other grey dot must appear as the $n-m$ grey dots with lowest weight in the right column.

In preparation for the discussion of (finitized) characters below, we associate a monomial $q^{w}$ to a double-column configuration where $w$ is the weight of the configuration. Here $q$ is a formal parameter but later it will be the modular parameter. We define the polynomial $\left\langle\begin{array}{c}M \\ m, n\end{array}\right\rangle_{q}$ as the sum of the monomials associated to the elements of $A_{m, n}^{M}$. In [24], we show that this polynomial admits the closed-form expression

$$
\left\langle\begin{array}{c}
M \\
m, n
\end{array}\right\rangle_{q}=q^{\frac{1}{2} m(m+1)+\frac{1}{2} n(n+1)}\left(\left[\begin{array}{c}
M \\
m
\end{array}\right]_{q}\left[\begin{array}{c}
M \\
n
\end{array}\right]_{q}-q^{n-m+1}\left[\begin{array}{c}
M \\
n+1
\end{array}\right]_{q}\left[\begin{array}{c}
M \\
m-1
\end{array}\right]_{q}\right)
$$

where $\left[\begin{array}{l}k \\ j\end{array}\right]_{q}$ is a $q$-binomial (Gaussian polynomial). These are generalized $q$-Narayana numbers [25] to which they coincide when $m=n$. Despite the minus sign, (3.32) is 
actually fermionic in the sense that it is a polynomial with only non-negative coefficients [24].

\subsection{Selection rules}

We now turn to the description of the selection rules. They depend on the parity of $N$. Based on a body of empirical data obtained by examining the double-row transfer matrices analytically as well as numerically, we make the following conjecture.

Selection rules For a system with $N$ columns and $\ell$ defects where $N-\ell \equiv 0$ mod 2 , the selection rules are equivalent to singling out the following sets of admissible double-column configurations

$$
N \text { even : } \bigcup_{m=0}^{\frac{N-\ell}{2}}\left(A_{m, m+\frac{\ell-2}{2}}^{\frac{N-2}{2}} \cup A_{m, m+\frac{\ell}{2}}^{\frac{N-2}{2}}\right), \quad N \text { odd }: \bigcup_{m=0}^{\frac{N-\ell}{2}} A_{m, m+\frac{\ell-1}{2}}^{\frac{N-1}{2}}
$$

By construction, all these unions are disjoint unions. It is noted that the case where

$\ell=0$ is special since $A_{m, m-1}^{\frac{N-2}{2}}=\emptyset$. As argued below, $\ell=0$ gives rise to the identity field in the associated CFT.

\section{Finite-Size Corrections}

The partition function of critical dense polymers on a lattice of $N$ columns and $M$ double rows is defined by

$$
Z_{N, M}=\operatorname{Tr} \boldsymbol{D}(u)^{M}=\sum_{n} D_{n}(u)^{M}=\sum_{n} e^{-M E_{n}(u)}
$$

where the sum is over all eigenvalues of $\boldsymbol{D}(u)$ including possible multiplicities and $E_{n}(u)$ is the energy associated to the eigenvalue $D_{n}(u)$. A refinement of (4.1) to treat explicitly sectors with different numbers of defects is given in the next section. Conformal invariance of the model in the continuum scaling limit would dictate [26, 27] that the leading finitesize corrections for large $N$ are of the form

$$
E_{n}(u)=-\ln D_{n}(u) \simeq 2 N f_{b u l k}+f_{b d y}+\frac{2 \pi \sin 2 u}{N}\left(-\frac{c}{24}+\Delta+k\right)
$$

Here $f_{b u l k}$ is the bulk free energy per face, $f_{b d y}$ is the boundary or surface free energy, while $c$ is the central charge of the CFT whose spectrum of conformal weights is given by the possible values of $\Delta$ with excitations or descendants labelled by the non-negative integers $k$. As the analysis below will confirm, the asymptotic behaviour of the eigenvalues (3.20) are in accordance with (4.2). 
The logarithms of the eigenvalues (3.20) involves sums of terms which are singular in the limit $N \rightarrow \infty$. To remedy this, one can introduce the function

$$
F(t)=\ln \left(\frac{t}{\sin t}+t \sin 2 u\right)=\ln t+\ln \left(\frac{1}{\sin t}+\sin 2 u\right)
$$

which is well defined as $t \rightarrow 0$ and where the $u$ dependence for simplicity has been formally suppressed. This trick was employed in a similar analysis [22] of the Ising model, and it allows us to examine the asymptotic behaviour of the logarithms. Indeed, the Euler-Maclaurin formula now gives

$$
\sum_{k=0}^{m} F(a+k h) \simeq \frac{1}{h} \int_{a}^{b} F(t) d t+\frac{1}{2}[F(b)+F(a)]+\frac{h}{12}\left[F^{\prime}(b)-F^{\prime}(a)\right]
$$

where $b=a+m h$, and due to the split (4.3), we also need to approximate the logarithm of the gamma function

$$
\ln \Gamma(y) \simeq\left(y-\frac{1}{2}\right) \ln y-y+\frac{1}{2} \ln (2 \pi)+\frac{1}{12 y}
$$

As a partial evaluation of the energies (4.2), we find

$$
\ln \prod_{j}\left(\frac{1}{\sin t_{j}}+\epsilon_{j} \sin 2 u\right) \simeq \sum_{j}\left(F\left(t_{j}\right)-\ln t_{j}\right)-2 \sin 2 u \sum_{j \in \mathcal{E}} t_{j}
$$

where $\mathcal{E}$ is the subset of $j$ indices for which $\epsilon_{j}=-1$ and $\mathcal{E}$ remains finite as $N \rightarrow \infty$. Likewise, $\mathcal{M}$ denotes the subset of $j$ indices for which $\mu_{j}=-1$ and it also remains finite as $N \rightarrow \infty$. The argument $t_{j}$ is defined in (3.22). We finally find

$$
\begin{array}{rlr}
E_{n}(u) \simeq 2 N f_{b u l k}+f_{b d y} & \\
+\frac{2 \pi \sin 2 u}{N} \begin{cases}\left(\frac{2}{24}+\sum_{j \in \mathcal{E}_{N, n}} j+\sum_{j \in \mathcal{M}_{N, n}} j\right), & N \text { even } \\
\left(\frac{2}{24}-\frac{1}{8}+\sum_{j \in \mathcal{E}_{N, n}}\left(j-\frac{1}{2}\right)+\sum_{j \in \mathcal{M}_{N, n}}\left(j-\frac{1}{2}\right)\right), & N \text { odd }\end{cases}
\end{array}
$$

In these expressions, the bulk free energy per face is given by

$$
f_{\text {bulk }}=\ln \sqrt{2}-\frac{1}{\pi} \int_{0}^{\pi / 2} \ln \left(\frac{1}{\sin t}+\sin 2 u\right) d t
$$

whereas the boundary free energy is given by

$$
f_{b d y}=\ln (1+\sin 2 u)
$$




\section{Conformal Field Theory}

The next task and our main objective is to calculate analytically the conformal data associated with our model of critical dense polymers. By comparing the expressions (4.7) with the finite-size corrections (4.2), the central charge is readily seen to be

$$
c=-2
$$

while the lowest conformal weights for given parity of $N$ are $\Delta=0$ and $\Delta=-\frac{1}{8}$, respectively. The full spectrum of conformal weights and their excitations are discussed in the following.

\subsection{Finitized characters}

It is recalled that the matrix representation of the double-row transfer matrix depends on which space $\boldsymbol{D}(u)$ is acting. Since the action of $\boldsymbol{D}(u)$ on $\mathcal{L}_{N}$ is upper block-triangular, the partition function (4.1) may be written

$$
Z_{N, M}=\sum_{s} \sum_{n} D_{n}^{(s)}(u)^{M}=\sum_{s} \sum_{n} e^{-M E_{n}^{(s)}(u)}
$$

where $D_{n}^{(s)}(u)$ and $E_{n}^{(s)}(u)$ are the eigenvalues and associated energies obtained by restricting to the $\ell=s-1$ sector. Likewise, information on the excitations or descendants are contained in the appropriate sets $\mathcal{E}_{N, n}^{(s)}$ and $\mathcal{M}_{N, n}^{(s)}$, cf. (4.7). Here we have introduced the extended Kac label $s$ related to the number of defects $\ell$ by

$$
s=\ell+1
$$

Now, the link between the lattice model and the characters of the CFT is governed by the modular parameter $q$ defined by

$$
q=e^{-2 \pi \tau}, \quad \tau=\frac{M}{N} \sin 2 u
$$

The ratio $M / N$ is the aspect ratio and $\vartheta=2 u$ is the anisotropy angle related to the geometry of the lattice. As already discussed, the spectrum of the CFT is extracted from the eigenvalues, that is, the specification of $\mathcal{E}_{N, n}^{(s)}$ and $\mathcal{M}_{N, n}^{(s)}$ for all $s$. This amounts to determining appropriate selection rules. The relevant rules are stated explicitly in (3.33) where $\ell=s-1$.

For finite $N$, we thus find that the full spectrum can be organized in finitized characters $\chi_{s}^{(N)}(q)$ and we find that they are given by

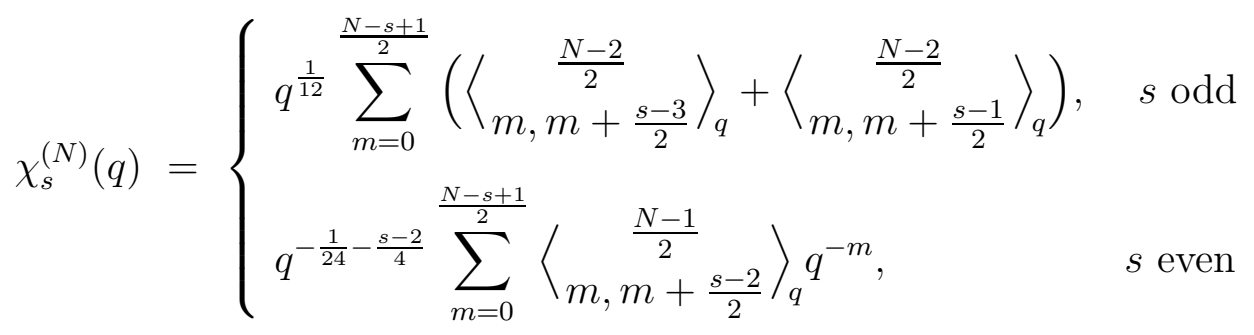


where some terms may vanish, cf. (3.30). It is emphasized that these are fermionic character expressions.

In [24], we prove that the finitized characters (5.5) can be written collectively as

$$
\chi_{s}^{(N)}(q)=q^{-\frac{c}{24}+\Delta_{s}}\left(\left[\begin{array}{c}
N \\
\frac{N-s+1}{2}
\end{array}\right]_{q}-q^{s}\left[\begin{array}{c}
N \\
\frac{N-s-1}{2}
\end{array}\right]_{q}\right)
$$

where

$$
\Delta_{s}=\frac{s^{2}-4 s+3}{8}, \quad s \in \mathbb{Z}_{>}
$$

This corresponds to the first column of the extended Kac table

$$
\begin{array}{|c|}
\hline \vdots \\
\hline \frac{15}{8} \\
\hline 1 \\
\hline \frac{3}{8} \\
\hline 0 \\
\hline-\frac{1}{8} \\
\hline 0 \\
\hline
\end{array}
$$

Setting, $\Delta_{s}=\Delta_{1, s}$, this agrees with the general $\mathcal{L} \mathcal{M}\left(p, p^{\prime}\right)$ formula

$$
\Delta_{r, s}=\frac{\left(r p^{\prime}-s p\right)^{2}-\left(p^{\prime}-p\right)^{2}}{4 p p^{\prime}}
$$

with $\left(p, p^{\prime}\right)=(1,2)$ for critical dense polymers. This explicitly confirms the form of the finitized characters in [7] for the case $\left(p, p^{\prime}\right)=(1,2)$ with $r=1$. Finitized characters like (5.6) were introduced first in [28, 29].

By construction, a finitized character must contain information on the dimensionality of the vector space of states. Here this is reflected in the formula

$$
\lim _{q \rightarrow 1} \chi_{s}^{(N)}(q)=\operatorname{dim} \mathcal{L}_{N, s-1}
$$

\subsection{Spectrum of $c=-2$ CFT}

In the continuum scaling limit, the finitized characters carry over to the full characters of the associated CFT. We have already identified the central charge $c=-2$ and the conformal weights (5.7) of this CFT while the associated characters are given by

$$
\chi_{s}(q)=\lim _{N \rightarrow \infty} \chi_{s}^{(N)}(q)=\frac{q^{\frac{(s-2)^{2}}{8}}}{\eta(q)}\left(1-q^{s}\right)
$$


where the Dedekind eta function is defined by

$$
\eta(q)=q^{\frac{1}{24}} \prod_{m=1}^{\infty}\left(1-q^{m}\right)
$$

The character $\chi_{s}(q)$ is recognized as the Virasoro character of a particular quasirational highest-weight representation of the Virasoro algebra. To appreciate this, we recall that the Verma module $V_{r, s}$ of a Virasoro highest-weight representation of highest weight (5.9) is defined for all $r, s \in \mathbb{Z}_{>}$and $p, p^{\prime}$ two coprime positive integers. The associated quotient module

$$
Q_{r, s}=V_{r, s} / V_{r,-s}
$$

is also defined for every pair of positive integers $r, s$ and corresponds to a quasi-rational representation. The character of such a quasi-rational representation is given by

$$
\chi_{r, s}(q)=\frac{q^{\frac{1-c}{24}}}{\eta(q)}\left(q^{\Delta_{r, s}}-q^{\Delta_{r,-s}}\right)=\frac{q^{\frac{1-c}{24}}}{\eta(q)} q^{\Delta_{r, s}}\left(1-q^{r s}\right)
$$

where $c=1-6 \frac{\left(p^{\prime}-p\right)^{2}}{p p^{\prime}}$. Critical dense polymers correspond to $\left(p, p^{\prime}\right)=(1,2)$ in which case $\chi_{1, s}(q)=\chi_{s}(q)$, as already indicated.

So far for critical dense polymers, from the lattice approach, we have only identified sectors with $r=1$. The associated quasi-rational characters correspond to representations $(1, s)$ which are only irreducible for $s=1$ or $s$ even. From the point of view of CFT, one conventionally works with irreducible representations as the natural building blocks. This is indeed the case in [30] where the complete set of irreducible representations necessarily involve sectors corresponding to $r>1$, even after identification of irreducible representations with identical characters following the classification of irreducible characters in the appendix of [7]. A lattice analysis of the set of irreducible representations will appear elsewhere.

A variety of character identities exist for the set of quasi-rational representations. In particular, every quasi-rational character (5.14) with $r>1$ can be written as a linear combination of characters with $r=1$

$$
\chi_{1+k, s}(q)=\sum_{j=0}^{k} \chi_{s+4 j-2 k}(q)
$$

where it is implicit that

$$
\chi_{0}(q)=0, \quad \chi_{-s}(q)=-\chi_{s}(q)
$$

This extends to general $r, s$ and coprime $p, p^{\prime}$ [24] by admitting linear combinations of the $p$ left-most columns in the extended Kac table

$$
\chi_{r+k p, s}(q)=\sum_{j=0}^{k} \chi_{r, s+(2 j-k) p^{\prime}}(q)+\sum_{j=0}^{k-1} \chi_{p-r, s+(2 j+1-k) p^{\prime}}(q)
$$

employing a straightforward extension of (5.15). It is emphasized, though, that these character identities are blind to any Jordan cells, cf. Section 7. 


\section{Hamiltonian Limit}

The Hamiltonian limit of the double-row transfer matrix $\boldsymbol{D}(u)$ is defined in the planar algebra as the $N$-tangle appearing as the leading non-trivial term in an expansion with respect to $u$, that is,

$$
\boldsymbol{D}(u)=\boldsymbol{I}-2 u \boldsymbol{H}+\mathcal{O}\left(u^{2}\right)
$$

It follows that

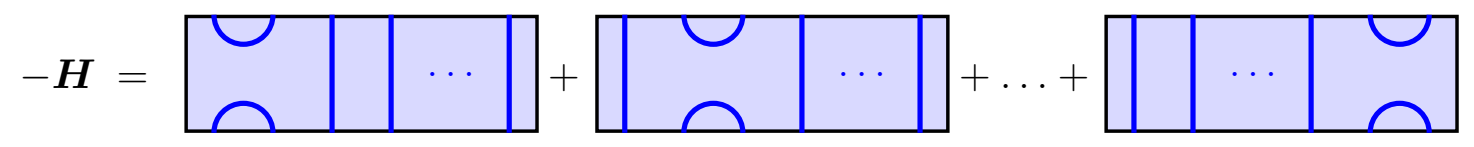

which in terms of the generators of the linear TL algebra merely corresponds to

$$
\boldsymbol{H}=-\sum_{j=1}^{N-1} e_{j}
$$

The set of normalized eigenvectors of $\boldsymbol{H}$ is the same as that of $\boldsymbol{D}(u)$ while the eigenvalues of $\boldsymbol{H}$ and $\boldsymbol{D}(u)$ are different. The conformal spectra are nevertheless the same as we will see below.

As preparation for the discussion of fusion in Section 7, it is useful to adjust this definition of $\boldsymbol{H}$ to the various sectors. We thus introduce the $s$-dependent Hamiltonian $\boldsymbol{H}_{(1, s)}$ by augmenting the $N$-tangles in (6.2) by the identity $(s-1)$-tangle, that is,

$$
\boldsymbol{H}_{(1, s)}=\begin{array}{|lr|l|l|}
\hline \boldsymbol{H}_{(1,1)} & \vdots & & \ldots \\
\hline
\end{array}
$$

where $\boldsymbol{H}_{(1,1)}=\boldsymbol{H}$. This augmented Hamiltonian acts naturally on link states with $N+s-1$ nodes (and zero defects) of which the $s-1$ right-most nodes, called boundary nodes, must be connected to nodes of the original $N$-tangle Hamiltonian, called bulk nodes. Likewise, a resulting state where a pair of boundary nodes are connected, is set to zero. The vertical dashed line in (6.4) indicates the separation into bulk and boundary parts. The spectrum of $\boldsymbol{H}_{(1, s)}$ is identical to the spectrum of $\boldsymbol{H}$ when the latter is restricted to acting on the $(1, s)$ sector.

We will renormalize the Hamiltonian $\boldsymbol{H}_{(1, s)}$ by shifting the 'ground state energy' to facilitate the introduction of the finitized dilatation generator and to make the relation to the finitized characters more transparent. This also ensures that the renormalized Hamiltonian $\mathcal{H}_{(1, s)}$ is non-negative definite. We define it by

$$
\mathcal{H}_{(1, s)}=\boldsymbol{H}_{(1, s)}+2\left(\sum_{j=1}^{\left\lfloor\frac{N-1}{2}\right\rfloor} \sin t_{j}\right) \boldsymbol{I}=\boldsymbol{H}_{(1, s)}+ \begin{cases}\frac{\sqrt{2} \sin \frac{(N-2) \pi}{4 N}}{\sin \frac{\pi}{2 N}} \boldsymbol{I}, & s \text { odd } \\ \frac{2 \sin ^{2} \frac{(N-1) \pi}{4 N}}{\sin \frac{\pi}{2 N}} \boldsymbol{I}, & s \text { even }\end{cases}
$$


where $t_{j}$ is defined in (3.22), and find that its eigenvalues are given by

$$
2 \sum_{j \in \mathcal{E}_{N}^{(s)}} \sin t_{j}+2 \sum_{j \in \mathcal{M}_{N}^{(s)}} \sin t_{j}
$$

Since the eigenvalues of $\mathcal{H}_{(1, s)}$ can be expressed as the linear combinations (6.6) of terms like $\sin t_{j}$, we may now define the finitized dilatation generator $L_{0}^{(1, s)}$ by replacing these summands $\sin t_{j}$ by $N t_{j} / 2 \pi$ in the Jordan canonical form of $\mathcal{H}_{(1, s)}-\frac{1+(-1)^{s}}{16} \boldsymbol{I}$ when the diagonal entries of the latter are expressed as in (6.6). That is, we are defining the finitized dilatation generator by the formal replacement

$$
L_{0}^{(1, s)}=\left.\operatorname{Jordan}\left(\mathcal{H}_{(1, s)}-\frac{1+(-1)^{s}}{16} \boldsymbol{I}\right)\right|_{\sin t_{j} \mapsto \frac{N t_{j}}{2 \pi}}
$$

The dependence on $N$ has been suppressed. An eventual off-diagonal part of this Jordan canonical form is unaltered by this replacement. We observe, though, that the Hamiltonian $\mathcal{H}_{(1, s)}$ is diagonalizable. This is in accordance with our observation in Section 3.3 that the double-row transfer matrix $\boldsymbol{D}(u)$ itself is diagonalizable when restricted to the sector $(1, s)$. In the discussion of fusion in Section 7 , on the other hand, we will encounter Hamiltonians which are not diagonalizable.

It also follows that the eigenvalues of $L_{0}^{(1, s)}$ are

$$
\begin{array}{cc}
\sum_{j \in \mathcal{E}_{N}^{(s)}} j+\sum_{j \in \mathcal{M}_{N}^{(s)}} j, & s \text { odd } \\
-\frac{1}{8}+\sum_{j \in \mathcal{E}_{N}^{(s)}}\left(j-\frac{1}{2}\right)+\sum_{j \in \mathcal{M}_{N}^{(s)}}\left(j-\frac{1}{2}\right), & s \text { even }
\end{array}
$$

in accordance with (4.7). This confirms the assertion above that the double-row transfer matrix and the Hamiltonian have the same conformal spectra. We can also re-express the finitized characters as

$$
\chi_{1, s}^{(N)}(q)=\operatorname{Tr} q^{L_{0}^{(1, s)}-c / 24}
$$

thus mimicking the definition of the ordinary Virasoro characters.

\section{Fusion}

In this section, we explain the diagrammatic implementation of fusion within our framework [7]. Although we focus here on critical dense polymers and the sectors with $r=1$ (labelled by $s=1,2, \ldots$ ), the construction extends to the other logarithmic minimal models and sectors with $r>1$, albeit with more involved computations. For convenience in the description of our fusion prescription, we work with the Hamiltonian limit of the double-row transfer matrix. The conclusions about fusion are the same but more tedious to reach when the analysis is based on the double-row transfer matrix $\boldsymbol{D}(u)$ itself. 


\subsection{Fusion prescription}

To study the fusion of the representations $(1, s)$ and $\left(1, s^{\prime}\right)$, we consider a system of size $N$ augmented by $s-1$ auxiliary nodes to the left and $s^{\prime}-1$ auxiliary nodes to the right. That is, the corresponding Hamiltonian is

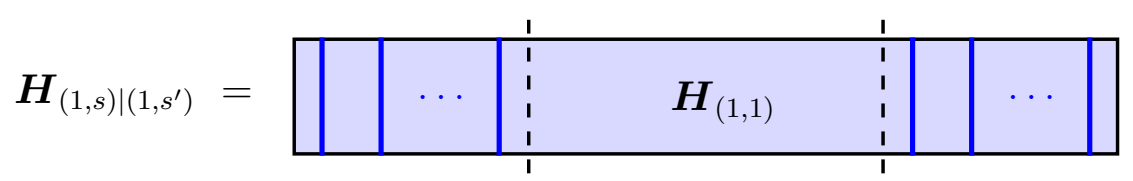

where $N \geq s+s^{\prime}-2$ and $N-s-s^{\prime}=0 \bmod 2$. It acts naturally on link states with $N+s+s^{\prime}-2$ nodes and zero defects, though not all such link states are allowed. Our fusion prescription excludes the link states where a pair of nodes from the same boundary are linked. This means, in particular, that a left-boundary node must be linked to either a right-boundary node or a bulk node but not to another left-boundary node. Suppressing the dependence on $N$, this set of link states is denoted $\mathcal{L}_{(1, s) \mid\left(1, s^{\prime}\right)}$.

Letting $h$ denote the number of half-arcs connecting nodes on the left boundary with nodes on the right boundary, the space $\mathcal{L}_{(1, s) \mid\left(1, s^{\prime}\right)}$ is naturally decomposed as the disjoint union of the spaces $\mathcal{L}_{(1, s) \mid\left(1, s^{\prime}\right)}^{(h)}$ containing link states with fixed $h$, that is,

$$
\mathcal{L}_{(1, s) \mid\left(1, s^{\prime}\right)}=\mathcal{L}_{(1, s) \mid\left(1, s^{\prime}\right)}^{\left(\min \left(s, s^{\prime}\right)-1\right)} \cup \cdots \cup \mathcal{L}_{(1, s) \mid\left(1, s^{\prime}\right)}^{(1)} \cup \mathcal{L}_{(1, s) \mid\left(1, s^{\prime}\right)}^{(0)}
$$

Viewed from the bulk, links to the left boundary are equivalent to links to the right boundary as they are all just defects. We may therefore re-interpret the states in $\mathcal{L}_{(1, s) \mid\left(1, s^{\prime}\right)}^{(h)}$ as states in $\mathcal{L}_{(1,1) \mid\left(1, s+s^{\prime}-1-2 h\right)}^{(0)}=\mathcal{L}_{\left(1, s+s^{\prime}-1-2 h\right)}$. Explicitly, for the case $(1,4) \mid(1,3)$ with $N=7$ and $h=1$, we can map state-by-state as follows. First we move the first node from the left to the right boundary carrying its link (shown dashed and in blue in the illustration (7.3) below) with it in a planar fashion. We repeat this with the second and third nodes on the left so that now all boundary nodes are on the right. Finally, we remove the $h$ spectator half-arcs (in this case, the single one shown dashed and in blue) on the right boundary which are common to all allowed link states. This two-step process is illustrated here

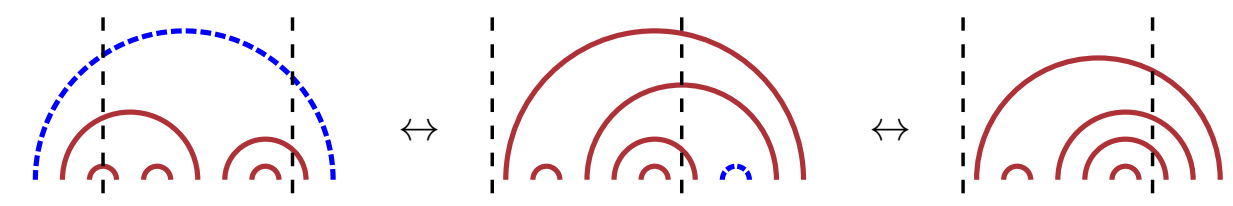

This re-interpretation naively implies the $\operatorname{sl}(2)$ fusion rule

$$
\left(1, s_{1}\right) \otimes_{f}\left(1, s_{2}\right)=\bigoplus_{s}(1, s)
$$

where the sum is over $s=\left|s_{1}-s_{2}\right|+1,\left|s_{1}-s_{2}\right|+3, \ldots, s_{1}+s_{2}-1$. Care must be exercised, though, since defects can be annihilated in pairs. Indeed, this latter property 
implies that the action of the Hamiltonian on (7.2) is upper block-triangular rather than block-diagonal. There is thus the possibility of forming indecomposable representations in which case the right side of (7.4) is not a direct sum. In the following, we will characterize these representations and conjecture the fusion rules applying to the complete set of representations generated by fusion of the $(1, s)$ building blocks.

Due to the decomposition (17.2), the renormalization of the Hamiltonian $\boldsymbol{H}_{(1, s) \mid\left(1, s^{\prime}\right)}$ is defined as in (6.5) by

$$
\mathcal{H}_{(1, s) \mid\left(1, s^{\prime}\right)}=\boldsymbol{H}_{(1, s) \mid\left(1, s^{\prime}\right)}+ \begin{cases}\frac{\sqrt{2} \sin \frac{(N-2) \pi}{4 N}}{\sin \frac{\pi}{2 N}} \boldsymbol{I}, & s+s^{\prime} \text { even } \\ \frac{2 \sin ^{2} \frac{(N-1) \pi}{4 N}}{\sin \frac{\pi}{2 N}} \boldsymbol{I}, & s+s^{\prime} \text { odd }\end{cases}
$$

Due to the decomposition (7.2) and the subsequent re-interpretation, the eigenvalues of $\mathcal{H}_{(1, s) \mid\left(1, s^{\prime}\right)}$ are

$$
2 \sum_{j \in \mathcal{E}_{N}^{\left(s+s^{\prime}-1-2 h\right)}} \sin t_{j}+2 \sum_{j \in \mathcal{M}_{N}^{\left(s+s^{\prime}-1-2 h\right)}} \sin t_{j}, \quad h=0, \ldots, \min \left(s, s^{\prime}\right)-1
$$

The associated finitized dilatation generator thus reads

$$
L_{0}^{(1, s) \mid\left(1, s^{\prime}\right)}=\left.\operatorname{Jordan}\left(\mathcal{H}_{(1, s) \mid\left(1, s^{\prime}\right)}-\frac{1-(-1)^{s+s^{\prime}}}{16} \boldsymbol{I}\right)\right|_{\sin t_{j} \mapsto \frac{N t_{j}}{2 \pi}}
$$

and has eigenvalues

$$
\begin{aligned}
& \sum_{j \in \mathcal{E}_{N}^{\left(s+s^{\prime}-1-2 h\right)}} j+\sum_{j \in \mathcal{M}_{N}^{\left(s+s^{\prime}-1-2 h\right)}} j, \quad h=0, \ldots, \min \left(s, s^{\prime}\right)-1, \quad s+s^{\prime} \text { even } \\
& -\frac{1}{8}+\sum_{j \in \mathcal{E}_{N}^{\left(s+s^{\prime}-1-2 h\right)}}\left(j-\frac{1}{2}\right)+\sum_{j \in \mathcal{M}_{N}^{\left(s+s^{\prime}-1-2 h\right)}}\left(j-\frac{1}{2}\right), \quad h=0, \ldots, \min \left(s, s^{\prime}\right)-1, \quad s+s^{\prime} \text { odd }
\end{aligned}
$$

\subsection{Indecomposable representations}

As a first example, we consider the fusion

$$
(1,2) \otimes_{f}(1,2)=(1,1) \oplus_{i}(1,3)
$$

in some detail. The subscript $i$ refers to the indecomposable structure of the right side. Let the system size be $N=4$ in which case

$$
\begin{aligned}
& \mathcal{L}_{(1,2) \mid(1,2)}^{(1)}=\{\overbrace{1}^{\prime}, n_{1}^{\prime}, 1\}
\end{aligned}
$$

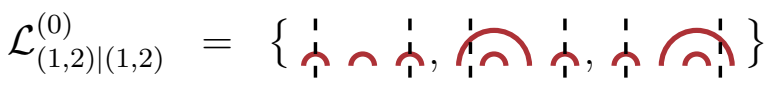


Acting on these link states listed in the order indicated here, the matrix representation of the Hamiltonian $\boldsymbol{H}_{(1,2) \mid(1,2)}$ is

$$
\boldsymbol{H}_{(1,2) \mid(1,2)}=-\left(\begin{array}{ccccc}
0 & 2 & 0 & 1 & 1 \\
1 & 0 & 0 & 0 & 0 \\
0 & 0 & 0 & 1 & 1 \\
0 & 0 & 1 & 0 & 0 \\
0 & 0 & 1 & 0 & 0
\end{array}\right)
$$

The Jordan canonical form of the Hamiltonian $\mathcal{H}_{(1,2) \mid(1,2)}$ thus reads

$$
\operatorname{Jordan}\left(\mathcal{H}_{(1,2) \mid(1,2)}\right)=\operatorname{diag}\left[\left(\begin{array}{ll}
0 & 1 \\
0 & 0
\end{array}\right), 2 \sin \frac{\pi}{4},\left(\begin{array}{cc}
4 \sin \frac{\pi}{4} & 1 \\
0 & 4 \sin \frac{\pi}{4}
\end{array}\right)\right]
$$

in which case the finitized dilatation generator, after the replacement $\sin \frac{\pi}{4} \mapsto \frac{4 \frac{\pi}{4}}{2 \pi}=\frac{1}{2}$, becomes

$$
L_{0}^{(1,2) \mid(1,2)}=\operatorname{diag}\left[\left(\begin{array}{ll}
0 & 1 \\
0 & 0
\end{array}\right), 1,\left(\begin{array}{ll}
2 & 1 \\
0 & 2
\end{array}\right)\right]
$$

Also for $N=4$, the finitized dilatation generators associated to $\boldsymbol{H}_{(1,1)}$ and $\boldsymbol{H}_{(1,3)}$ are likewise found to be

$$
L_{0}^{(1,1)}=\operatorname{diag}[0,2], \quad L_{0}^{(1,3)}=\operatorname{diag}[0,1,2]
$$

This means that the finitized partition function associated to (7.11) decomposes as

$$
\begin{aligned}
Z_{(1,2) \mid(1,2)}^{(4)}(q)=\chi_{(1,1)}^{(4)}(q)+\chi_{(1,3)}^{(4)}(q) & =q^{1 / 12}\left[\left(1+q^{2}\right)+\left(1+q+q^{2}\right)\right] \\
& =q^{1 / 12}\left(2+q+2 q^{2}\right)
\end{aligned}
$$

in accordance with the fusion rule (7.9) $)$.

The decomposition (7.15) does not contain information on the rank-two Jordan cells appearing in (7.13) as the characters only reflect the diagonal part. The non-trivial Jordan-cell structure is a finite-size manifestation of the right side of (7.9) being indecomposable. It is observed that a Jordan cell is formed whenever possible and we have verified for $N=2,4,6,8$ that this property persists. It is also noted that the Hamiltonian is upper block-triangular meaning that states contributing to $\chi_{3}(q)$ can be mapped to states contributing to $\chi_{1}(q)$ but not vice versa. These observations are compatible with the properties of the indecomposable representation $\mathcal{R}_{1,1}$, appearing in [30], with character $\chi_{1}(q)+\chi_{3}(q)$. Assuming equivalence of the indecomposable representations, we will adopt their notation here and henceforth denote the right side of (7.9) by $\mathcal{R}_{1,1}$.

As a second example, we consider the fusion

$$
(1,2) \otimes_{f}(1,4)=(1,3) \oplus_{i}(1,5)
$$


Let the system size be $N=6$ in which case

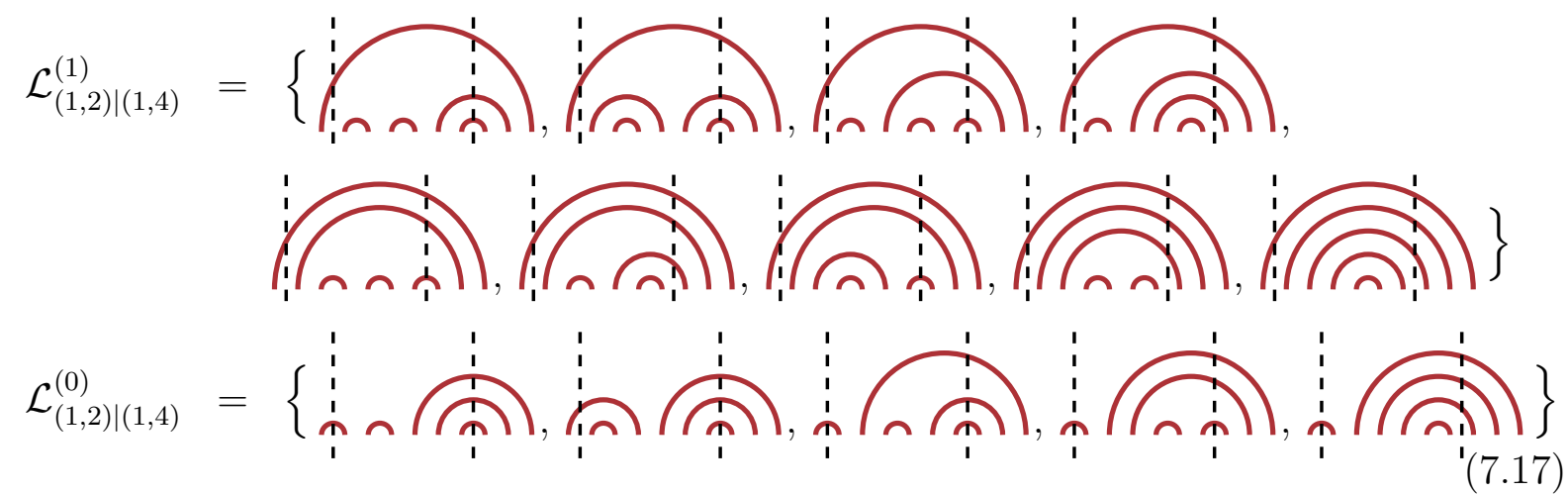

Acting on these link states listed in the order indicated here, the matrix representation of the Hamiltonian $\boldsymbol{H}_{(1,2) \mid(1,4)}$ is

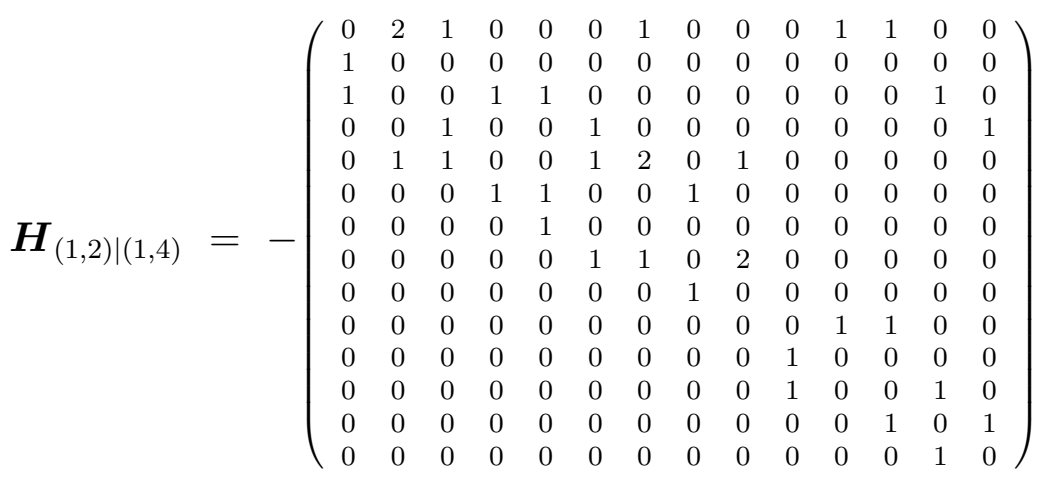

The Jordan canonical form of the associated renormalized Hamiltonian $\mathcal{H}_{(1,2) \mid(1,4)}$ thus reads

$$
\begin{aligned}
\operatorname{Jordan}\left(\mathcal{H}_{(1,2) \mid(1,4)}\right)= & \operatorname{diag}\left[0,\left(\begin{array}{ll}
1 & 1 \\
0 & 1
\end{array}\right),\left(\begin{array}{cc}
\sqrt{3} & 1 \\
0 & \sqrt{3}
\end{array}\right), 2,1+\sqrt{3}, 1+\sqrt{3}, 2 \sqrt{3}\right. \\
& \left.\left(\begin{array}{cc}
2+\sqrt{3} & 1 \\
0 & 2+\sqrt{3}
\end{array}\right),\left(\begin{array}{cc}
1+2 \sqrt{3} & 1 \\
0 & 1+2 \sqrt{3}
\end{array}\right), 2+2 \sqrt{3}\right]
\end{aligned}
$$

where we have used that $2 \sin \frac{\pi}{6}=1$ and $2 \sin \frac{\pi}{3}=\sqrt{3}$. The associated finitized dilatation generator is now found to be

$$
L_{0}^{(1,2) \mid(1,4)}=\operatorname{diag}\left[0,\left(\begin{array}{ll}
1 & 1 \\
0 & 1
\end{array}\right),\left(\begin{array}{ll}
2 & 1 \\
0 & 2
\end{array}\right), 2,3,3,4,\left(\begin{array}{ll}
4 & 1 \\
0 & 4
\end{array}\right),\left(\begin{array}{ll}
5 & 1 \\
0 & 5
\end{array}\right), 6\right]
$$

It is observed that the repeated eigenvalue 3 does not form a Jordan cell. This is unlike the fusion (7.9) above where a Jordan cell is formed whenever possible. Also for $N=6$, the finitized dilatation generators associated to $\boldsymbol{H}_{(1,3)}$ and $\boldsymbol{H}_{(1,5)}$ are likewise found to be

$$
L_{0}^{(1,3)}=\operatorname{diag}[0,1,2,2,3,4,4,5,6], \quad L_{0}^{(1,5)}=\operatorname{diag}[1,2,3,4,5]
$$


This means that the finitized partition function associated to (7.18) decomposes as

$$
\begin{aligned}
Z_{(1,2) \mid(1,4)}^{(6)}(q) & =\chi_{(1,3)}^{(6)}(q)+\chi_{(1,5)}^{(6)}(q) \\
& =q^{1 / 12}\left[\left(1+q+2 q^{2}+q^{3}+2 q^{4}+q^{5}+q^{6}\right)+q\left(1+q+q^{2}+q^{3}+q^{4}\right)\right] \\
& =q^{1 / 12}\left(1+2 q+3 q^{2}+2 q^{3}+3 q^{4}+2 q^{5}+q^{6}\right)
\end{aligned}
$$

and we note that the 'missing' Jordan cell corresponds to $q^{3}$. Continuing our comparison with the results in [30], we denote the associated indecomposable representation $(1,3) \oplus_{i}$ $(1,5)$ by $\mathcal{R}_{2,1}$.

Based on the examples above and many other explicit evaluations of fusions of the form $(1, s) \otimes_{f}\left(1, s^{\prime}\right)$, we have observed that indecomposable representations are only formed by the combinations

$$
\mathcal{R}_{j, 1}=(1,2 j-1) \oplus_{i}(1,2 j+1), \quad j=1,2, \ldots
$$

and that they arise as the result of the fusions

$$
(1,2) \otimes_{f}(1,2 j)=(1,2 j-1) \oplus_{i}(1,2 j+1)
$$

The characters and indecomposable structure of these indecomposable representations correspond to the definition of $\mathcal{R}_{j, 1}$ in [30]. In particular, the off-diagonal part of the Jordan decomposition of the Hamiltonian or dilatation generator maps $(1,2 j+1)$ to $(1,2 j-1)$ but not the other way. It is stressed that the Jordan cells rendering the representations $\mathcal{R}_{j, 1}$ indecomposable all have rank two. As we will argue in the following, no new indecomposable representations arise when considering fusions of indecomposable representations. The set $\left\{\mathcal{R}_{j, 1}\right\}$ is also the complete set of indecomposable representations appearing in [30].

Here we can refine the characterization of the indecomposable representation $\mathcal{R}_{j, 1}$. Indeed, with reference to the fermionic expressions for the finitized characters $\chi_{(1,2 j-1)}^{(N)}(q)$ and $\chi_{(1,2 j+1)}^{(N)}(q)$ in (5.5) $)$, we have found that in the cases examined,

$$
\begin{aligned}
\chi_{\mathcal{R}_{j, 1}}^{(N)}(q)= & \chi_{(1,2 j-1)}^{(N)}(q)+\chi_{(1,2 j+1)}^{(N)}(q) \\
= & \left.q^{\frac{1}{12}}\left(\sum_{m=0}^{\frac{N-2 j+2}{2}}\left\langle m, \frac{N-2}{2}\right\rangle_{m}\right\rangle_{q}+\sum_{m=0}^{\frac{N-2 j}{2}}\langle m, m+j-1\rangle_{q}\right) \\
& \quad+q^{\frac{N-2}{12}}\left(\sum_{m=0}^{\frac{N-2 j}{2}}\langle m, m+j-1\rangle_{q}+\sum_{m=0}^{\frac{N-2}{2}}\left\langle\frac{\frac{N-2}{2}}{m, j}+j\right\rangle_{q}\right)
\end{aligned}
$$

Here the arrow indicates the off-diagonal action of the dilatation generator which maps every state in the given sum to its identical mirror state in the target sum. No other offdiagonal action is present. Together with the statement that the map is from $(1,2 j+1)$ 
to $(1,2 j-1)$ only, this gives a full description of the indecomposable structure of (the finitized version of) $\mathcal{R}_{j, 1}$. It is noted that the number of Jordan cells appearing in the finitized version of $\mathcal{R}_{j, 1}$ is given by

$$
\lim _{q \rightarrow 1} \sum_{m=0}^{\frac{N-2 j}{2}}\left\langle\begin{array}{c}
\frac{N-2}{2} \\
m, m+j-1
\end{array}\right\rangle_{q}=\left(\begin{array}{c}
N-1 \\
\frac{N-2 j}{2}
\end{array}\right)-\left(\begin{array}{c}
N-1 \\
\frac{N+2 j}{2}
\end{array}\right)=\lim _{q \rightarrow 1} \chi_{1,2 j}^{(N-1)}(q)
$$

We find it useful to view the indecomposable structure of $\mathcal{R}_{j, 1}$ as the following formal four-dimensional matrix containing a single rank-two Jordan cell

$$
\begin{aligned}
& \operatorname{diag}\left[\sum_{m=0}^{\frac{N-2 j+2}{2}}\left\langle\begin{array}{c}
\frac{N-2}{2} \\
m, m+j-2
\end{array}\right\rangle_{q}\right. \\
& \left(\begin{array}{cc}
\sum_{m=0}^{\frac{N-2 j}{2}}\left\langle\begin{array}{c}
\frac{N-2}{2} \\
m+j-1\rangle_{q}
\end{array}\right. & 1 \\
0 & \sum_{m=0}^{\frac{N-2 j}{2}}\left\langle\begin{array}{c}
\frac{N-2}{2} \\
m, m+j-1
\end{array}\right\rangle_{q}
\end{array}\right), \\
& \left.\sum_{m=0}^{\frac{N-2 j-2}{2}}\left\langle\begin{array}{c}
\frac{N-2}{2} \\
m, m+j
\end{array}\right\rangle_{q}\right]
\end{aligned}
$$

It follows and is emphasized that if a Jordan cell corresponding to a particular energy (that is, power of $q$ in the associated finitized character (7.25)) is present for a particular system size, a Jordan cell corresponding to the same energy is present for all larger system sizes. This supports our claim that this particular Jordan-cell structure persists in the limit $N \rightarrow \infty$.

Let us reexamine the two fusions $(1,2) \otimes_{f}(1,2)$ and $(1,2) \otimes_{f}(1,4)$ and put into the perspective of (7.25) why a Jordan cell was observed whenever possible in the first of these fusions but not in the second. For finite system size $N$, the right side of the fusion (7.9) is described by (7.25) with $j=1$. Since $\left\langle\begin{array}{c}M \\ m, n\end{array}\right\rangle$ vanishes if $m>n$, cf. (3.32), the sum $\sum_{m=0}^{\frac{N-2 j+2}{2}}\left\langle\begin{array}{c}\frac{N-2}{2} \\ m, m+j-2\end{array}\right\rangle_{q}$ vanishes and a Jordan cell is formed whenever possible. Also for finite $N$, the right side of the fusion (7.16) is described by (7.25) with $j=2$. In this case, a Jordan cell is only formed for the matching sums while the remaining two sums in general will involve states with equal energies. This was illustrated in (7.19) for $N=6$ in which case the two sums in question read

$$
\left.\sum_{m=0}^{\frac{N-2 j+2}{2}}\left\langle\begin{array}{c}
\frac{N-2}{2} \\
m, m+j-2
\end{array}\right\rangle_{q}\right|_{N=6, j=2}=1+q^{2}+q^{3}+q^{4}+q^{6},\left.\quad \sum_{m=0}^{\frac{N-2 j-2}{2}}\left\langle\begin{array}{c}
\frac{N-2}{2} \\
m, m+j
\end{array}\right\rangle_{q}\right|_{N=6, j=2}=q^{3}
$$


This explains why the Jordan cell corresponding to $q^{3}$ was 'missing'.

We wish to point out that one can be misled when considering very small system sizes $N$. As this example illustrates

$$
\chi_{\mathcal{R}_{j, 1}}^{(2 j-2)}(q)=q^{\frac{1}{2}+\Delta_{1,2 j-1}}=\chi_{(1,2 j-1)}^{(2 j-2)}(q)
$$

an ambiguity due to finite-size effects can arise since the indecomposable structure of $\mathcal{R}_{j, 1}$ is only visible if $N$ is big enough to accommodate it.

\subsection{Fusion rules}

Our next task is to examine fusion of indecomposable representations. We thus need a characterization of the boundary conditions corresponding to $\mathcal{R}_{j, 1}$. Since $\mathcal{R}_{j, 1}=(1,2 j-$ 1) $\oplus_{i}(1,2 j+1)$, the boundary should accommodate $2 j-2$ as well as $2 j$ boundary nodes. To preserve the number of nodes in the associated link states, the former case is represented by $2 j$ boundary nodes of which two must be linked together by a spectator half-arc. The right boundary associated to $\mathcal{R}_{j, 1}$ thus involves $2 j$ nodes and our fusion prescription now selects the following set of link states

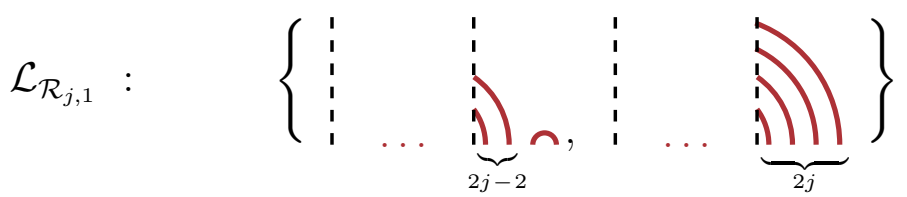

According to the discussion following the decomposition (7.2), we can simply define the (finitized version of the) indecomposable representation $\mathcal{R}_{j, 1}$ by imposing the boundary condition just introduced. This applies to the double-row transfer matrix $\boldsymbol{D}(u)$ as well as to the Hamiltonian limit thereof which can be described as in (6.4) by

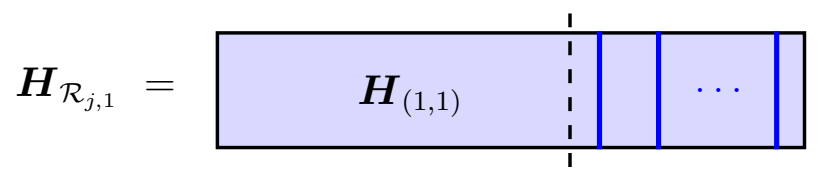

Here the bulk Hamiltonian has been augmented by the identity $2 j$-tangle and it is recapitulated that the boundary condition dictates that $\boldsymbol{H}_{\mathcal{R}_{j, 1}}$ acts on the link states (7.30) with no left-boundary nodes.

As a first example, we consider

$$
(1,3) \otimes_{f} \mathcal{R}_{1,1}=\mathcal{R}_{1,1} \oplus \mathcal{R}_{2,1}
$$


explicitly for $N=4$. The set of link states decomposes as

$$
\begin{aligned}
& \mathcal{L}_{(1,3) \mid \mathcal{R}_{1,1}}^{(2)}=\{\overbrace{1 \cap \Omega}^{\prime}, \overbrace{(2) !}^{\prime}\}
\end{aligned}
$$

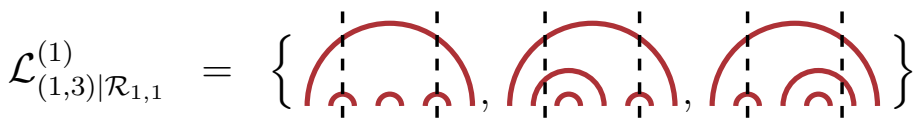

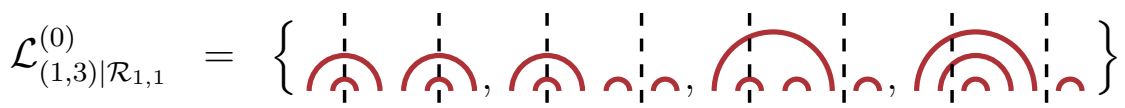

Acting on these link states listed in the order indicated here, the matrix representation of the Hamiltonian $\boldsymbol{H}_{(1,3) \mid \mathcal{R}_{1,1}}$ is

$$
\boldsymbol{H}_{(1,3) \mid \mathcal{R}_{1,1}}=-\left(\begin{array}{ccccccccc}
0 & 2 & 0 & 1 & 1 & 0 & 0 & 0 & 0 \\
1 & 0 & 0 & 0 & 0 & 0 & 0 & 0 & 0 \\
0 & 0 & 0 & 1 & 1 & 1 & 0 & 0 & 0 \\
0 & 0 & 1 & 0 & 0 & 0 & 0 & 0 & 0 \\
0 & 0 & 1 & 0 & 0 & 0 & 0 & 0 & 0 \\
0 & 0 & 0 & 0 & 0 & 0 & 0 & 0 & 0 \\
0 & 0 & 0 & 0 & 0 & 1 & 0 & 1 & 0 \\
0 & 0 & 0 & 0 & 0 & 0 & 1 & 0 & 1 \\
0 & 0 & 0 & 0 & 0 & 0 & 0 & 1 & 0
\end{array}\right)
$$

Following the outline above, we work out the finitized dilatation generator

$$
L_{0}^{(1,3) \mid \mathcal{R}_{1,1}}=\operatorname{diag}\left[0,\left(\begin{array}{ll}
0 & 1 \\
0 & 0
\end{array}\right), 1,\left(\begin{array}{ll}
1 & 1 \\
0 & 1
\end{array}\right), 2,\left(\begin{array}{ll}
2 & 1 \\
0 & 2
\end{array}\right)\right]
$$

as well as those associated to $\boldsymbol{H}_{\mathcal{R}_{1,1}}$ and $\boldsymbol{H}_{\mathcal{R}_{2,1}}$, that is,

$$
L_{0}^{\mathcal{R}_{1,1}}=\operatorname{diag}\left[\left(\begin{array}{ll}
0 & 1 \\
0 & 0
\end{array}\right), 1,\left(\begin{array}{ll}
2 & 1 \\
0 & 2
\end{array}\right)\right], \quad L_{0}^{\mathcal{R}_{2,1}}=\operatorname{diag}\left[0,\left(\begin{array}{ll}
1 & 1 \\
0 & 1
\end{array}\right), 2\right]
$$

In accordance with (7.32), we see that, up to a permutation operation,

$$
L_{0}^{(1,3) \mid \mathcal{R}_{1,1}}=\operatorname{diag}\left[L_{0}^{\mathcal{R}_{1,1}}, L_{0}^{\mathcal{R}_{2,1}}\right]
$$

in which case the finitized partition function decomposes as

$$
\begin{aligned}
Z_{(1,3) \mid \mathcal{R}_{1,1}}^{(4)}(q)=\chi_{\mathcal{R}_{1,1}}^{(4)}(q)+\chi_{\mathcal{R}_{2,1}}^{(4)}(q) & =q^{1 / 12}\left[\left(2+q+2 q^{2}\right)+\left(1+2 q+q^{2}\right)\right] \\
& =q^{1 / 12}\left(3+3 q+3 q^{2}\right)
\end{aligned}
$$

As a second example, we consider

$$
(1,2) \otimes_{f} \mathcal{R}_{1,1}=(1,2) \oplus(1,2) \oplus(1,4)
$$


illustrating that fusion of an indecomposable representation does not necessarily produce an indecomposable representation. We demonstrate this explicitly for $N=3$ in which case

$$
\begin{aligned}
& \mathcal{L}_{(1,2) \mid \mathcal{R}_{1,1}}^{(1)}=\{\overbrace{1}^{1}, \overbrace{1}^{1}
\end{aligned}
$$

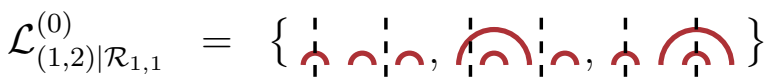

Even though these sets of link states appear the same as those in (7.10), the separation into bulk and boundary nodes is different. Acting on the link states (7.40) listed in the order indicated, the matrix representation of the Hamiltonian $\boldsymbol{H}_{(1,2) \mid \mathcal{R}_{1,1}}$ is

$$
\boldsymbol{H}_{(1,2) \mid \mathcal{R}_{1,1}}=-\left(\begin{array}{ccccc}
0 & 1 & 0 & 0 & 1 \\
1 & 0 & 0 & 0 & 0 \\
0 & 0 & 0 & 1 & 1 \\
0 & 0 & 1 & 0 & 0 \\
0 & 0 & 0 & 0 & 0
\end{array}\right)
$$

which is seen to be diagonalizable. The associated finitized partition function decomposes as

$$
Z_{(1,2) \mid \mathcal{R}_{1,1}}^{(3)}(q)=2 \chi_{(1,2)}^{(3)}(q)+\chi_{(1,4)}^{(3)}(q)=q^{1 / 12}\left[2\left(1+q^{2}\right)+q\right]=q^{1 / 12}\left(2+q+2 q^{2}\right)
$$

in accordance with (7.39).

As a third example, we consider

$$
\mathcal{R}_{1,1} \otimes_{f} \mathcal{R}_{1,1}=\mathcal{R}_{1,1} \oplus \mathcal{R}_{1,1} \oplus \mathcal{R}_{2,1}
$$

For $N=4$, the numbers of link states are

$$
\left|\mathcal{L}_{\mathcal{R}_{1,1} \mid \mathcal{R}_{1,1}}^{(2)}\right|=2, \quad\left|\mathcal{L}_{\mathcal{R}_{1,1} \mid \mathcal{R}_{1,1}}^{(1)}\right|=3, \quad\left|\mathcal{L}_{\mathcal{R}_{1,1} \mid \mathcal{R}_{1,1}}^{(0)}\right|=9
$$

based on which we have worked out the associated finitized dilatation generator

$$
L_{0}^{\mathcal{R}_{1,1} \mid \mathcal{R}_{1,1}}=\operatorname{diag}\left[0,\left(\begin{array}{ll}
0 & 1 \\
0 & 0
\end{array}\right),\left(\begin{array}{ll}
0 & 1 \\
0 & 0
\end{array}\right), 1,1,\left(\begin{array}{ll}
1 & 1 \\
0 & 1
\end{array}\right), 2,\left(\begin{array}{ll}
2 & 1 \\
0 & 2
\end{array}\right),\left(\begin{array}{ll}
2 & 1 \\
0 & 2
\end{array}\right)\right]
$$

The generators $L_{0}^{\mathcal{R}_{1,1}}$ and $L_{0}^{\mathcal{R}_{2,1}}$ for $N=4$ are given in (7.36). In accordance with (7.43), we find that, up to a permutation operation,

$$
L_{0}^{\mathcal{R}_{1,1} \mid \mathcal{R}_{1,1}}=\operatorname{diag}\left[L_{0}^{\mathcal{R}_{1,1}}, L_{0}^{\mathcal{R}_{1,1}}, L_{0}^{\mathcal{R}_{2,1}}\right]
$$

yielding the following decomposition of the associated finitized partition function

$$
Z_{\mathcal{R}_{1,1} \mid \mathcal{R}_{1,1}}^{(4)}(q)=2 \chi_{\mathcal{R}_{1,1}}^{(4)}(q)+\chi_{\mathcal{R}_{2,1}}^{(4)}(q)
$$


Based on these examples and many others, we conjecture the following set of fusion rules.

Fusion rules The fusion algebra of the representations $(1, s)$ and $\mathcal{R}_{j, 1}$ where $s, j=$ $1,2,3, \ldots$ is given by

$$
\begin{aligned}
\left(1,2 j_{1}\right) \otimes_{f}\left(1,2 j_{2}\right) & =\bigoplus_{j} \mathcal{R}_{j, 1} \\
\left(1,2 j_{1}\right) \otimes_{f} \mathcal{R}_{j_{2}, 1} & =\bigoplus_{j}[(1,2 j-2) \oplus(1,2 j) \oplus(1,2 j) \oplus(1,2 j+2)] \\
\mathcal{R}_{j_{1}, 1} \otimes_{f} \mathcal{R}_{j_{2}, 1} & =\bigoplus_{j}\left[\mathcal{R}_{j-1,1} \oplus \mathcal{R}_{j, 1} \oplus \mathcal{R}_{j, 1} \oplus \mathcal{R}_{j+1,1}\right] \\
\left(1,2 j_{1}-1\right) \otimes_{f}\left(1,2 j_{2}-1\right) & =\bigoplus_{j=\left|j_{1}-j_{2}\right|+1}^{j_{1}+j_{2}-1}(1,2 j-1) \\
\left(1,2 j_{1}-1\right) \otimes_{f}\left(1,2 j_{2}\right) & =\bigoplus_{j=\left|j_{1}-j_{2}-\frac{1}{2}\right|+\frac{1}{2}}^{j_{1}-j_{2}-1}(1,2 j) \\
\left(1,2 j_{1}-1\right) \otimes_{f} \mathcal{R}_{j_{2}, 1} & =\bigoplus_{j=\left|j_{1}-j_{2}-\frac{1}{2}\right|+\frac{1}{2}}^{\mathcal{R}_{j, 1}}
\end{aligned}
$$

where $(1,0)=\mathcal{R}_{0,1}=0$. The three unspecified sums are over

$$
j=\left|j_{1}-j_{2}\right|+1,\left|j_{1}-j_{2}\right|+3, \ldots, j_{1}+j_{2}-1
$$

whereas the three specified sums are with unit increments.

As expected, $(1,1)$ is seen to play the role of the identity representation. It is noted that the set of representations $(1, s)$ and $\mathcal{R}_{j, 1}$ is closed under fusion. Several distinct fusion subalgebras and ideals are easily identified. It is also noted that the fourth and fifth fusion rules are identical to (7.4) with $s_{1}$ odd.

We stress that we have considered fusion generated from the $(1, s)$ representations and that they are only irreducible for $s=1$ or $s$ even. The fusion rules in the CFT approach [30], on the other hand, are expressed in terms of the irreducible representations $\mathcal{V}_{j, 1}$ and $\mathcal{V}_{j, 2}$ only and the indecomposable representations resulting from fusion. A discussion of the irreducible representations from the lattice point of view will appear elsewhere.

Consistent with the fusion rules of [30, we have observed that our fusion rules (7.48) are reproduced by a simple extension by linearity, namely

$$
(1,2 j) \sim \mathcal{V}_{j, 2}, \quad(1,2 j-1) \sim \mathcal{V}_{j-1,1}+\mathcal{V}_{j, 1}, \quad j=1,2, \ldots
$$

where $\mathcal{V}_{0,1}=0$. We have already identified the indecomposable representations $\mathcal{R}_{j, 1}$ with those appearing in [30]. 


\section{Conclusion}

In this paper, we have solved exactly a model of critical dense polymers on strips of finite width. This has been achieved, without invoking an $n \rightarrow 0$ or $Q \rightarrow 0$ limit, by solving directly our model of critical dense polymers within the framework of YangBaxter integrability. The calculations have been carried out for an infinite family of $(1, s)$ integrable boundary conditions which impose $\ell=s-1$ defects in the bulk. Our study of the physical combinatorics, which is the classification of the physical states using combinatorial objects, has revealed some interesting connections to $q$-Narayana numbers and natural generalizations thereof. Using the planar TL algebra, Yang-Baxter techniques and functional equations in the form of inversion identities, we have been able to calculate analytically the bulk and boundary free energies, the central charge $c=-2$, the conformal weights $\Delta_{s}=\frac{(2-s)^{2}-1}{8}$ and conformal characters in accord with previous results [3, 4, 5, 6]. More particularly, since we were able to solve the model exactly for finite system sizes, we have explicitly confirmed the finitized conformal characters proposed in [7].

In support of our claim that the CFT associated with critical dense polymers is logarithmic, we have shown in explicit examples how fusion of our $(1, s)$ boundary conditions in some cases lead to indecomposable representations $\mathcal{R}_{j, 1}$. We have argued, by implementing fusion diagrammatically, that fusion among the $(1, s)$ representations and indecomposable representations $\mathcal{R}_{j, 1}$ closes, and we have conjectured the general form of the associated fusion rules. A proper comparison with the fusion algebra of Gaberdiel and Kausch [30], however, requires that our fusion algebra is formulated in terms of irreducible representations alongside the indecomposable representations. A discussion of these irreducible representations from the lattice point of view will appear elsewhere. We have nevertheless found that the fusion rules presented here are in accordance with those in [30] in the sense that our fusion rules can be obtained from theirs by extending by linearity to the $(1, s)$ representations.

Again, it is a well-known abstract result [19] that the TL algebra admits indecomposable representations at roots of unity. We emphasize that our motivation here is to construct explicit lattice Hamiltonians for these representations in the case of critical dense polymers, to relate them to physical boundary conditions and to understand their fusion properties in physical terms.

To claim a complete understanding of our model of critical dense polymers, we should study its extension from the strip to other topologies. Most importantly, the model should be examined on the geometric cylinder and torus where the effects of non-contractible loops will play a role. This constitutes work in progress.

\section{Acknowledgments}

This work is supported by the Australian Research Council. The authors thank JeanBernard Zuber for discussions. 


\section{References}

[1] P.G. de Gennes, Phys. Rev. Lett. A38 (1972) 339; Scaling Concepts in Polymer Physics, Cornell University, Ithaca (1979); J. des Cloizeaux, J. Phys. (Paris) 36 (1975) 281.

[2] C.M. Fortuin, P.W. Kasteleyn, Physica 57 (1972) 536; F.Y. Wu, Rev. Mod. Phys. 54 (1982) 235.

[3] H. Saleur, J. Phys. A20 (1987) 455-470; J. Phys. A19 (1986) L807-L810; Phys. Rev. B35 (1987) 3657-3660.

[4] B. Duplantier, J. Phys. A19 (1986) L1009-L1014.

[5] H. Saleur, Nucl. Phys. B382 (1992) 486-531.

[6] N. Read, H. Saleur, Nucl. Phys. B613 (2001) 409.

[7] P.A. Pearce, J. Rasmussen, J.-B. Zuber, J. Stat. Mech. (2006) P11017.

[8] B. Duplantier, F. David, J. Stat. Phys. 51 (1988) 327-434.

[9] A. Sedrakyan, Nucl. Phys. B554 (1999) 514-536.

[10] M.R. Gaberdiel, H.G. Kausch, Phys. Lett. B386 (1996) 131-137.

[11] B.L. Feigin, A.M. Gainutdinov, A.M. Semikhatov, I.Yu. Tipunin, Nucl. Phys. B757 (2006) 303-343; Kazhdan-Lusztig dual quantum group for logarithmic extensions of Virasoro models, hep-th/0606506 (2006).

[12] M.R. Gaberdiel, I. Runkel, J. Phys. A39 (2006) 14745-14780.

[13] H.G. Kausch, Nucl. Phys. B583 (2000) 513-541.

[14] S. Mahieu, P. Ruelle, Phys. Rev. E64 (2001) 066130; P. Ruelle, Phys. Lett. B539 (2002) 172-177; G. Piroux, P. Ruelle, J. Stat. Mech. 0410 (2004) P005; J. Phys. A38 (2005) 1451-1472; Phys. Lett. B607 (2005) 188-196.

[15] N.S. Izmailian, V.B. Priezzhev, P. Ruelle, C.-K. Hu, Phys. Rev. Lett. 95 (2005) 260602.

[16] J.L. Jacobsen, N. Read, H. Saleur, Phys. Rev. Lett. 93 (2004) 038701.

[17] E.V. Ivashkevich, C.-K. Hu, Phys. Rev. E71 (2005) 015104 (R).

[18] V.F.R. Jones, Planar algebras I, math.QA/9909027. 
[19] P.P. Martin, Potts models and related problems in statistical mechanics, Series on Advances in Statistical Mechanics, Volume 5, World Scientific, Singapore (1991).

[20] J.L. Cardy, Nucl. Phys. B270 (1986) 186; Nucl. Phys. B275 (1986) 200; H. Saleur, M. Bauer, Nucl. Phys. B320 (1989) 591-624; J.L. Cardy, Nucl. Phys. B324 (1989) 581-596; R.E. Behrend, P.A. Pearce, J.-B. Zuber, J. Phys. A31 (1998) L763-L770; R.E. Behrend, P.A. Pearce, V.B. Petkova, J.-B. Zuber, Nucl. Phys. B579 (2000) 707-773; R.E. Behrend, P.A. Pearce, J. Stat. Phys. 102 (2001) 577-640.

[21] R.J. Baxter Exactly solved models in statistical mechanics (London, 1982) Academic Press.

[22] D.L. O’Brien, P.A. Pearce, S.O. Warnaar, Physica A228 (1996) 63-77.

[23] R.E. Behrend, P.A. Pearce, D.L. O’Brien, J. Stat. Phys. 84 (1996) 1-48.

[24] P.A. Pearce, J. Rasmussen, Physical combinatorics of critical dense polymers, in preparation (2006).

[25] J. Fürlinger, J. Hofbauer, J. Combin. Theory A40 (1985) 248-264; P. Brändén, Discrete Math. 281 (2004) 67-81.

[26] H.W.J. Blöte, J.L. Cardy, M.P. Nightingale, Phys. Rev. Lett. 56 (1986) 742-745.

[27] I. Affleck, Phys. Rev. Lett. 56 (1986) 746-748.

[28] E. Melzer, Int. J. Mod. Phys. A9 (1994) 1115.

[29] A. Berkovich, Nucl. Phys. B431 (1994) 315.

[30] M.R. Gaberdiel, H.G. Kausch, Nucl. Phys. B477 (1996) 293-318. 